OPEN ACCESS

Edited by:

Mi-Hyeon Jang,

Rutgers, The State University of New Jersey, United States

Reviewed by: Qing-Feng Wu,

Chinese Academy of Sciences (CAS),

China

Anna Badner,

Stanford University, United States André F. Rendeiro,

Cornell University, United States

*Correspondence:

Elizabeth D. Kirby

Kirby.224@osu.edu

tPresent address:

Logan A. Walker,

Biophysics Program,

University of Michigan, Ann Arbor, MI, United States

Altan Turkoglu,

Department of Electrical \& Computer

Engineering,

OSU College of Engineering,

Columbus, $\mathrm{OH}$, United States

Specialty section:

This article was submitted to

Brain Disease Mechanisms,

a section of the journal

Frontiers in Molecular Neuroscience

Received: 07 November 2021 Accepted: 05 January 2022 Published: 31 January 2022

\section{Robust Transcriptional Profiling and Identification of Differentially Expressed Genes With Low Input RNA Sequencing of Adult Hippocampal Neural Stem and Progenitor Populations}

Jiyeon K. Denninger ${ }^{1}$, Logan A. Walker2t, Xi Chen ${ }^{3}$, Altan Turkoglu2t, Alex Pan ${ }^{3}$, Zoe Tapp ${ }^{4}$, Sakthi Senthilvelan', Raina Rindani', Olga N. Kokiko-Cochran ${ }^{4,5}$, Ralf Bundschuh ${ }^{2,6,7}$, Pearlly Yan ${ }^{3,6}$ and Elizabeth D. Kirby ${ }^{1,5 *}$

\footnotetext{
${ }^{1}$ Department of Psychology, College of Arts and Sciences, The Ohio State University, Columbus, OH, United States, ${ }^{2}$ Department of Physics, College of Arts and Sciences, The Ohio State University, Columbus, OH, United States,

${ }^{3}$ Comprehensive Cancer Center, College of Medicine, The Ohio State University, Columbus, OH, United States, ${ }^{4}$ Department of Neuroscience, Institute for Behavioral Medicine Research, The Ohio State University, Columbus, OH, United States, ${ }^{5}$ Chronic Brain Injury Program, The Ohio State University, Columbus, OH, United States, ${ }^{6}$ Division of Hematology, Department of Internal Medicine, College of Medicine, The Ohio State University, Columbus, OH, United States, ${ }^{7}$ Department of Chemistry and Biochemistry, College of Arts and Sciences, The Ohio State University, Columbus, $\mathrm{OH}$, United States
}

Multipotent neural stem cells (NSCs) are found in several isolated niches of the adult mammalian brain where they have unique potential to assist in tissue repair. Modern transcriptomics offer high-throughput methods for identifying disease or injury associated gene expression signatures in endogenous adult NSCs, but they require adaptation to accommodate the rarity of NSCs. Bulk RNA sequencing (RNAseq) of NSCs requires pooling several mice, which impedes application to labor-intensive injury models. Alternatively, single cell RNAseq can profile hundreds to thousands of cells from a single mouse and is increasingly used to study NSCs. The consequences of the low RNA input from a single NSC on downstream identification of differentially expressed genes (DEGs) remains insufficiently explored. Here, to clarify the role that low RNA input plays in NSC DEG identification, we directly compared DEGs in an oxidative stress model of cultured NSCs by bulk and single cell sequencing. While both methods yielded DEGs that were replicable, single cell sequencing using the 10X Chromium platform yielded DEGs derived from genes with higher relative transcript counts compared to non-DEGs and exhibited smaller fold changes than DEGs identified by bulk RNAseq. The loss of high fold-change DEGs in the single cell platform presents an important limitation for identifying disease-relevant genes. To facilitate identification of such genes, we determined an RNA-input threshold that enables transcriptional profiling of NSCs comparable to standard bulk sequencing and used it to establish a workflow for in vivo profiling of endogenous NSCs. We then applied this workflow to identify DEGs after 
lateral fluid percussion injury, a labor-intensive animal model of traumatic brain injury. Our work joins an emerging body of evidence suggesting that single cell RNA sequencing may underestimate the diversity of pathologic DEGs. However, our data also suggest that population level transcriptomic analysis can be adapted to capture more of these DEGs with similar efficacy and diversity as standard bulk sequencing. Together, our data and workflow will be useful for investigators interested in understanding and manipulating adult hippocampal NSC responses to various stimuli.

Keywords: adult neural stem cell, hippocampus, differential gene expression, single cell RNA sequencing, bulk RNA sequencing

\section{INTRODUCTION}

The subgranular zone (SGZ) of the hippocampal dentate gyrus (DG) is a unique neurogenic niche in the adult mammalian brain (Vicidomini et al., 2020; Denoth-Lippuner and Jessberger, 2021). Neural stem cells (NSCs) in the SGZ give rise to functional new neurons throughout adulthood that contribute to hippocampal memory and affect regulation and could also be a source for endogenous tissue repair after injury or disease (McAvoy and Sahay, 2017; Miller and Sahay, 2019). Over the past decade, transcriptional analysis using high-throughput RNA sequencing (RNAseq) technology has dramatically expanded knowledge of NSC molecular characteristics. For example, studies using prospectively identified stem and progenitor populations have uncovered previously unknown cell lineage relationships (Llorens-Bobadilla et al., 2015; Dulken et al., 2017; Baser et al., 2019; Berg et al., 2019). Other studies using more unbiased approaches have revealed regional and even cell-specific transcriptional differences or transcriptional changes during development, adult neurogenesis, or aging (Shin et al., 2015; Artegiani et al., 2017; Yuzwa et al., 2017; Hochgerner et al., 2018; Zywitza et al., 2018; Dulken et al., 2019; Mizrak et al., 2019). As studies of the NSC transcriptome expand, researchers are faced with an increasing variety of options for how to accomplish transcriptional profiling of this small, but critical, cell population.

Current major challenges to transcriptional profiling of NSCs include their relative sparsity in vivo and their transcriptional similarity to astrocytes. Both of these challenges have made bulk RNAseq of prospectively isolated NSCs a less attractive approach as it requires large cell number input and prospective isolation of the desired population. Instead, single cell RNAseq (scRNAseq) has emerged as the preferred technique to begin overcoming the above barriers. This approach uses the very small amounts of RNA present in single cells to generate thousands of individual cell transcriptomes with massively paralleled sequencing. scRNAseq studies of the adult mouse SVZ and SGZ have identified rare subpopulations of cells, as well as dynamic changes in gene expression at different developmental stages, maturation states, and regional locations (Llorens-Bobadilla et al., 2015; Shin et al., 2015; Artegiani et al., 2017). Several studies have adapted analytical methods from standard bulk sequencing to accommodate the technical challenges presented by using such low input to profile cells from other lineages (Robinson and Oshlack, 2010; Law et al., 2014;
Love et al., 2014). In addition, many pioneering studies in other cell types have also developed novel processing and analysis tools specifically designed to facilitate detection of cell heterogeneity or chronological mapping of developmental trajectories using scRNAseq transcriptomes (Trapnell et al., 2013; Finak et al., 2015; Shin et al., 2015; Qiu et al., 2017).

While these recent studies show that scRNAseq is a powerful approach to characterize differences between individual cells, it is not yet clear how effective it is for uncovering populationlevel changes in gene expression. Identification of differentially expressed genes (DEGs) induced by variables like injury or gene expression manipulation is critical to understanding the mechanisms underlying NSC function in both disease models and in healthy brains. It seems logical that the low input of scRNAseq would affect DEG discovery compared to standard bulk RNAseq, where DEG analysis in transcriptomics was first developed (Bhargava et al., 2015; Arzalluz-Luque et al., 2017). Investigation of this possible pitfall is still sparse. A small handful of studies has addressed robustness of different statistical analysis approaches for DEG identification, but some have contradictory findings (Ziegenhain et al., 2017; Soneson and Robinson, 2018; Wang et al., 2019; Mou et al., 2020; Squair et al., 2021). For example, Squair et al. (2021) suggest that scRNAseq DEGs are rife with false positives while Soneson and Robinson (2018) show that the most common statistical tests are quite resistant to false positives in scRNAseq DEG analysis. Most studies of this nature are retrospective-taking advantage of samples not originally processed for the purpose of identifying overlap and error in methods. Here, we directly compare scRNAseq (using the $10 \times$ Chromium platform) with bulk level RNAseq of cultured NSCs in a model of oxidative stress in vitro specifically to evaluate DEG identification across sequencing approaches. We found little overlap in DEGs identified by scRNAseq and bulk RNAseq, despite using the same source samples. While subsequent experiments showed that DEGs from both approaches were replicable and that our single cell analysis was resistant to false positives, we found that scRNAseq identified DEGs among genes that show a more moderate fold change and high relative transcript count when compared to the bulk RNAseq approach. Because many studies of DEGs would specifically benefit from identification of higher fold change transcripts which are more moderately expressed, we adapted and validated a limiting cell (lc) RNAseq approach for sequencing DG NSCs isolated from individual adult mice with similar reliability as more bulk 
RNAseq-like approaches. We further demonstrate the utility of this method by applying it to transcriptome profiling of NSCs and their intermediate progenitor cell (IPC) progeny from single adult mouse hippocampi after a lateral fluid percussion injury (LFPI) model of traumatic brain injury (TBI).

\section{MATERIALS AND METHODS}

\section{Animals}

Nestin-GFP mice (Mignone et al., 2004) (Jackson Labs, Bar Harbor, ME, United States, \#033927) and Wt C57Bl/6J mice (Jackson Labs, \#000664) were housed in a $12 \mathrm{~h}$ light-dark cycle with food and water ad libitum. To isolate brains for fluorescence in situ hybridization, adult mice (6-9 weeks old) were anesthetized with an intraperitoneal injection of ketamine $(87.5 \mathrm{mg} / \mathrm{kg})$ and xylazine $(12.5 \mathrm{mg} / \mathrm{kg})$. Mice were then transcardially perfused with ice cold PBS followed by cold $4 \%$ PFA. For immunofluorescence and whole DG RNA isolation, mice were only perfused with ice cold PBS. For lcRNAseq experiments, mice were only perfused with ice cold HBSS without calcium or magnesium. This study was approved by the Institutional Animal Care and Use Committee (IACUC) at the Ohio State University in accordance with institutional and national guidelines.

\section{Dentate Gyrus Isolation and Fluorescence Activated Cell Sorting for RNAseq}

To isolate DGs for subsequent fluorescence activated cell sorting (FACS), 6-9 weeks old adult mice ( $n=3$ biological replicates per group) were anesthetized and perfused with HBSS as described above. Following perfusion, brains were removed and placed in cold HBSS on ice. To expose the hippocampus, brains were bisected along the midsagittal line and the cerebellum and diencephalic structures were removed. Under a dissection microscope (Zeiss), the DG was excised using a beveled syringe needle and placed in ice cold HBSS without calcium or magnesium. DGs were then mechanically dissociated with sterile scalpel blades before enzymatic dissociation with a pre-warmed papain (Roche 10108014001)/dispase (Stem Cell Technologies, Vancouver, Canada, 07913)/DNase (Stem Cell Technologies, Vancouver, Canada, NC9007308) (PDD) cocktail at $37^{\circ} \mathrm{C}$ for $20 \mathrm{~min}$. Afterward, the tissue was again mechanically disrupted by trituration for $1 \mathrm{~min}$. Dissociated cells were collected by centrifugation at $500 \mathrm{~g}$ for $5 \mathrm{~min}$ before resuspending in HBSS without calcium/magnesium. Cells were then filtered through a $35 \mu \mathrm{m}$ nylon filter before staining with fluorescent antibodies (Supplementary Table 5) on ice for $30 \mathrm{~min}$. During the last $10 \mathrm{~min}$ of staining, Hoechst dye was added for live/dead discrimination. All cells were washed twice following staining and immediately sorted as NSC or IPC populations based on fluorescent markers with the FACSAria III (BD Biosciences, Franklin Lakes, NJ, United States). CD31-, CD45-, O1-, and O4 negative live cells were designated as NSCs if double positive for GLAST and Nestin-GFP or intermediate progenitor cells
(IPCs) if GFP positive and GLAST negative (Mignone et al., 2004; Llorens-Bobadilla et al., 2015). Three technical replicates of 300 cells each were sorted from each individual mouse into $1.5 \mathrm{~mL}$ microcentrifuge tubes containing cell lysis buffer from the Clontech SMART-Seq HT (Takara, Kusatsu, Shiga, Japan) kit for direct cDNA synthesis and RNAseq library generation.

\section{Cell Culture}

Neural stem cells were isolated from adult DGs of C57Bl6/J mice as described in Babu et al. (2011). Two separate lines, one from 4 pooled $\mathrm{C} 57 \mathrm{Bl6} / \mathrm{J}$ male mice and one from 4 pooled C57Bl6/J female mice, were used in experiments between passage 5 and 15. NSCs were cultured on poly-D-lysine (Sigma, St. Louis, MO, United States) and laminin (Invitrogen, Waltham, MA, United States) coated plates in Neurobasal A media (Invitrogen, Waltham, MA, United States) with $1 \times$ B27 supplement without vitamin A (Gibco, Waltham, MA, United States), $1 \times$ glutamax (Invitrogen, Waltham, MA, United States) and $20 \mathrm{ng} / \mathrm{ml}$ each of EGF and FGF2 (Peprotech, East Windsor, NJ, United States). There were no inherent differences in morphology or proliferation between NSC cultures and both lines differentiated into neurons and glia upon culture in differentiation conditions, as we previously showed (Denninger et al., 2020). All cultures were verified to be mycoplasma-free. For oxidative stress experiments, NSCs ( $n=3$ biological replicates) were treated at $70 \%$ confluency with $500 \mu \mathrm{M} \mathrm{H}_{2} \mathrm{O}_{2}$ (Sigma, St. Louis, MO, United States) or equal volume of vehicle (PBS) for $24 \mathrm{~h}$. All cells were harvested with brief accutase treatment and one wash with HBSS for RNA isolation or scRNAseq on the $10 \times$ Chromium platform.

\section{RNAseq of Cultured Cells and Whole Dentate Gyrus}

RNA from 30,000 cultured adult NSCs or whole DGs were isolated with the Clontech Nucleospin RNA XS Plus isolation kit (Takara 740990.10) per manufacturer protocol. RNA quality (RNA Integrity Number or RIN) and quantity was assessed using Agilent BioAnalyzer RNA 6000 Pico Kit and the Invitrogen Qubit RNA HS Assay kit (Invitrogen, Waltham, MA, United States), respectively. All cultured samples used a RIN value of 10 while whole DG samples had RIN values over 8. RNA from cultured NSCs was serially diluted to 10-, 100-, and 1,000-pg for RNA input quantity studies. Whole DG libraries for bulk RNAseq were generated with the NEBNext Ultra II Directional RNA Library prep kit (New England Biolabs, Ipswitch, MA, United States). The Clontech SMART-Seq HT (Takara) kit was used for global preamplification of cultured NSCs and 20\% ( 60 cell input) of the 300 FACS-isolated NSC cell lysate for low input RNAseq. Library generation followed the manufacturer manual except the reagents were miniaturized to $1 / 5$ th of the protocol volume for 300 cell samples. The quality and the quantity of purified cDNAs was assessed prior to sequencing library generation and sequencing using the Nextera XT Kit and Nextera XT Index Kit v2 Set A (Illumina, San Diego, CA, United States) following manufacturer instructions except for the miniaturization of reagent volume to a quarter of listed volume. 
Purified library products were then used in HiSeq 4000 pairedend sequencing (Illumina, San Diego, CA, United States) to a depth of 15-20 million $2 \times 150$ bp clusters. FASTQ files generated for each library were trimmed using AdapterRemoval v2.2.0 (Schubert et al., 2016), ensuring that all sequencing adapters were removed and that the average quality score for each read was above Q20 (representing 1 in 100 Illumina base error rate). Reads which were aligned by HISAT2 v2.0.6 (Kim et al., 2015) against rRNA, mtDNA, or PhiX bacteriophage (Illumina spikein control) sequences, retrieved from NCBI RefSeq (O'Leary et al., 2016), were removed from each FASTQ file, as these do not represent gene expression signal. All remaining reads were aligned against the reference mouse genome GRCm38p4 with HISAT2. The resulting BAM alignment files were sorted and indexed before further analysis.

Alignments were quantified using the featureCounts utility from the Subread package v1.5.1 (Liao et al., 2013, 2014) in unstranded mode using GENCODE (Harrow et al., 2006, 2012) mouse gene reference version M14 in GTF format. Custom Python scripts were used to produce a formatted gene expression counts table from the raw output of featureCounts. RNAseq Quality metrics were derived using a modification of the QuaCRS quality control workflow (Kroll et al., 2014) which includes running RNA-SeQC v1.1.8.1 (DeLuca et al., 2012), FASTQC v0.11.5, and RSeQC v2.6.2 (Wang et al., 2012). Finally, coverage maps of each BAM file were derived using the Bedtools 'genomecov' utility v2.27.0 (Quinlan and Hall, 2010).

RNAseq coverage maps were processed with the CLEAR v1.0 (Walker et al., 2020) workflow to determine which genes were reliably quantified. In brief, for each transcript in the UCSC GRCm38 release (Kuhn et al., 2013), a parameter $\left(\mu_{i}\right)$ is calculated, which represents the positional mean of the reads covering the transcript, normalized to the length of each sequence. Sequential bins of $250 \mu_{i}$ values each, ordered by descending expression, are fit to a sum of two beta distributions (Gupta and Nadarajah, 2004) for the determination of two free parameters, which are thresholded to determine genes which "pass" CLEAR. Unless otherwise noted, only genes passing CLEAR in all samples are used for downstream analysis. DEGs were derived from these CLEAR-filtered RNAseq expression counts tables following the DESeq2 v1.20.0 protocol (Love et al., 2014) implemented in R v3.5.0. DEGs are reported with adjusted p-values in our (Supplementary Tables 2-4) based on an FDR $q$-value of $<0.05$. DEGs were processed with the pcaExplorer v2.6.0 (Marini and Binder, 2019) visualization package to produce principal component analysis (PCA) projections using the default settings on r-log transformed counts. For phenotypic marker comparisons of NSCs and IPCs, raw count data were downloaded from GSE95753 associated with Hochgerner et al. (2018). Transcript counts were used for RGLs, nIPCs and NBs from mice P120 and P132 days of age. Counts were averaged within the three separable replicates in that dataset to give an average count per cell for each replicate.

\section{0x Chromium scRNAseq}

30,000 NSCs were pooled from triplicate $\mathrm{H}_{2} \mathrm{O}_{2-}$ or vehicletreated cultures $(10,000$ cells/replicate). Of those, $\sim 20,000$ cells per treatment were loaded onto the $10 \times$ Genomics single cell sequencing platform using the standard kit. The $3^{\prime}$ RNA-seq library was sequenced using paired-end 150 bp approach on an Illumina HiSeq 4000 sequencer. Data from vehicle-treated cells was previously published in Denninger et al. (2020) and similar analysis was performed here, but on the combined data, including both vehicle-treated and $\mathrm{H}_{2} \mathrm{O}_{2}$-treated cells. CellRanger v3.0.2 (Zheng et al., 2017) was used to demultiplex, align, and deduplicate sequencing reads in BCL files. Singlecell data in feature-barcode matrices were then processed using Seurat v3.0.1's default pipeline (Butler et al., 2018; Stuart et al., 2019) to identify unsupervised cell clusters and generate a uniform manifold approximation and projection (UMAP) plot. In brief, cells were filtered to exclude multiplets and damaged cells by excluding cells with unique feature count $>2,500$ or $<1,000$. From $\sim 41,805$ cells loaded, 26,916 were recovered, yielding a net capture rate of $64.3 \%$ and an estimated multiplet rate of $10.4 \%$, both of which are within manufacturer expectations. Data were then $\log$ normalized with default scale factor of 10,000 . The Seurat FindVariableFeatures function was then applied, followed by linear transformation (ScaleData function). PCA was run on the scaled data, followed by FindNeighbors and FindClusters. UMAPs were then created using the RunUMAP function. DEGs defining clusters (regardless of treatment) and defining treatments (regardless of cluster) were generated using the FindAllMarkers function, which uses a default of Wilcoxon rank sum test, unless otherwise noted. Adjusted $P$-values are Bonferroni-corrected using all features in the dataset. For data analysis where cell cycle effects were reduced, cell cycle scores were generated using the CellCycleScoring function of Seurat and then data were scaled with either standard CellCycleScore or the difference between S and G2M CellCycleScore defined as a variable to regress out. $p_{\text {adj }}<0.05$ was considered significant in all cases.

\section{Quantitative Real Time PCR}

Cultured NSCs were lysed in culture plates and RNA was isolated with the Bio-Rad Aurum ${ }^{\mathrm{TM}}$ Total RNA Mini Kit according to the manufacturer protocol. Isolated RNA was quantified and assessed for quality using the BioTek Epoch Microplate Spectrophotometer. cDNA was synthesized with the Bio-Rad iScript $^{\mathrm{TM}}$ cDNA Synthesis Kit in the Thermo Fisher Applied Biosystems 2720 Thermal Cycler according to manufacturer protocol. Quantitative real time PCR (qRT-PCR) was performed in the Bio-Rad CFX96 Touch Real-Time PCR Detection System with Bio-Rad SsoAdvanced Universal SYBR Green Supermix and primers listed in Supplementary Table 5. $\Delta \Delta C t$ values generated with normalization to housekeeping gene Rpl7 then converted to fold change (relative to vehicle).

\section{Lateral Fluid Percussion Injury}

All surgical procedures were performed as previously described (Tapp et al., 2020). Briefly, 6-9-week-old mice were anesthetized with $4 \%$ isoflurane gas in an induction chamber for $4 \mathrm{~min}$. Mice were then positioned in a stereotaxic frame before making a sagittal incision to expose the cranium. Midway between bregma and lambda on the right parietal bone, a 3.0-mm craniectomy 
was trephined, leaving the intact dura mater exposed. A modified portion of a Leur-Loc syringe $(3.0-\mathrm{mm}$ inside diameter) was secured over the craniectomy site with cyanoacrylate adhesive. Mice were placed in their home cages on a heating pad to recover. Once mice resumed normal activity, mice were returned to the vivarium for $24 \mathrm{~h}$. The next day, mice were anesthetized with $4 \%$ isoflurane in an induction chamber for $4 \mathrm{~min}$. Using the modified Leur-Loc syringe, mice were connected to the fluid percussion injury device (Custom Design \& Fabrication, Standston, VA, United States). For mice designated to the TBI group, a prepositioned pendulum was released onto the end of the LFPI device to deliver a fluid pulse onto the exposed dura mater, inducing a moderate LFPI. Sham mice were attached to the LFPI device but did not receive a fluid pulse. The modified syringe and adhesive were removed following LFPI or sham treatment. The incision was stapled closed. All animals were placed on a heating pad and injury severity was assessed with the self-righting reflex test. After the subjects demonstrated the righting reflex, they were returned to their home cages on a heating pad. Four hours after injury, mice were perfused for either histology ( $n=5$ biological replicates per group), RNA isolation ( $n=3$ biological replicates per group), or FACS $(n=3$ biological replicates per group).

\section{Histology (IF and RNAscope)}

For identification of in vivo cell types, brains from Nestin-GFP mice were harvested and fixed overnight at $4^{\circ} \mathrm{C}$ in $4 \%$ PFA before overnight equilibration in $30 \%$ sucrose. Serial $40 \mu \mathrm{m}$ sections were rinsed in PBS before blocking with 1\% normal donkey serum (Jackson) and $0.3 \%$ Triton X-100 in PBS for $30 \mathrm{~min}$ at room temperature. Sections were incubated with primary antibodies (Supplementary Table 5) overnight at $4^{\circ} \mathrm{C}$ in blocking solution on an orbital shaker. The next day, sections were washed with PBS and incubated with fluorescently conjugated secondary antibodies diluted in blocking solution (Supplementary Table 5) for $2 \mathrm{~h}$ at room temperature and counterstained with Hoechst 33342 (1:2000) for nuclear visualization. Sections were then washed and mounted onto slides before coverslipping with ProLong Gold anti-fade solution (Molecular Probes). Slides were imaged in 1- $\mu \mathrm{m} z$-stacks on an LSM700 confocal microscope (Zeiss) with a $40 \times$ oil objective.

For RNAscope, $4 \mathrm{~h}$ after LFPI or sham injury, brains from Wt $\mathrm{C} 57 \mathrm{Bl} / 6 \mathrm{~J}$ mice ( $n=5$ per group) were harvested and fixed overnight at $4^{\circ} \mathrm{C}$ in $4 \%$ PFA before serial overnight equilibration in 10, 20, and 30\% sucrose. Fixed equilibrated tissue was snap frozen in OCT in a dry ice/100\% ethanol bath and stored at $-70^{\circ} \mathrm{C} .12 \mu \mathrm{m}$ cryosections, 1 section per slide, were prepared with a cryostat. Slides were stored at $-70^{\circ} \mathrm{C}$ with desiccant until staining. RNA in situ hybridization was performed with RNAscope Multiplex Fluorescent v2 Assay (Advanced Cell Diagnostics, Newark, CA, United States) according to manufacturer recommendations for using fixed frozen tissue samples with the following modifications to enable concurrent immunohistochemical staining. The pretreatment steps were replaced with a 15 min modified citrate buffer (Dako) antigen retrieval step in a steamer at $95^{\circ} \mathrm{C}$. To enable subsequent immunohistochemical staining, the protease III step was excluded. Probes for mouse Slc5a3 (ACD custom design NPR-0006102), mouse Serpina3n (ACD 430191), and mouse Timp1 (ACD 316841) RNA were hybridized to tissue prior to immunohistochemical staining for GFAP and SOX2 protein. Immunostaining for GFAP and SOX2 was conducted as described above with the following exceptions. Blocking was performed with $10 \%$ normal donkey serum in TBS-1\% BSA. Antibody incubations were performed in TBS-1\% BSA. All washes were performed with TBST. DAPI provided by the RNAscope Multiplex Fluorescent kit was used for nuclear counterstaining. Images were acquired with a Zeiss Axio Observer Z1 microscope with Apotome for optical sectioning using a $20 \times$ air objective. Full $z$-stacks were acquired for analysis. NSCs were identified based on SOX2 positivity and GFAP+ apical processes extending from the nucleus in $1 \mu \mathrm{m} z$-stack images from $n=5$ mice. IPCs were similarly identified based on SOX2 positivity but without GFAP+ apical processes. Ten cells for each cell type were randomly selected from the subgranular zones of the DGs of 5 mice per treatment group for a total of 50 cells per group for each gene. mRNA puncta for each of the 3 genes were counted manually throughout the depth of each cell nucleus and length of cell processes.

\section{Statistics}

Statistics were performed as described in each figure legend. $\chi^{2}$ contingency test was used to compare reproducibility of DEGs identified in cultured NSCs after oxidative stress by scRNAseq and $1 \mathrm{ng}$ RNAseq. For the qRT-PCR confirmation of top DEGs by platform, a Mann-Whitney test was performed to identify individual genes with significant upregulation. MannWhitney tests were also used to compare the rank of DEG and non-DEG genes in cultured NSCs and in vivo NSCs/IPCs. To compare coefficient of variation between samples with or without CLEAR filtering, a one-way ANOVA with the Kruskal-Wallis test followed by Dunn's multiple comparisons or unpaired $t$-tests were applied. NSC and IPC mRNA expression per cell using each cell as a replicate ( $n=50$ per treatment group) of Slc5a3, Serpina3n, and Timp1 were compared using Mann-Whitney tests. mRNA expression per cell was also averaged for each mouse to compare gene expression differences with a biological replicate of $n=5$ using unpaired $t$-tests. $\chi^{2}$ contingency, Mann-Whitney, unpaired $t$-test, Dunn's multiple comparison, and ANOVA with the Kruskal-Wallis tests were performed using Prism (v9.0; GraphPad Software, LaJolla, CA, United States) and $p<0.05$ was considered significant.

\section{RESULTS}

\section{Differentially Expressed Genes Identified by 10x Chromium scRNAseq Versus Bulk Population Level RNAseq Are Different but Accurate}

To compare detection of differential gene expression by scRNAseq and bulk RNAseq of cultured cells, we used an in vitro model of oxidative stress with adult mouse DG-derived NSCs. We 


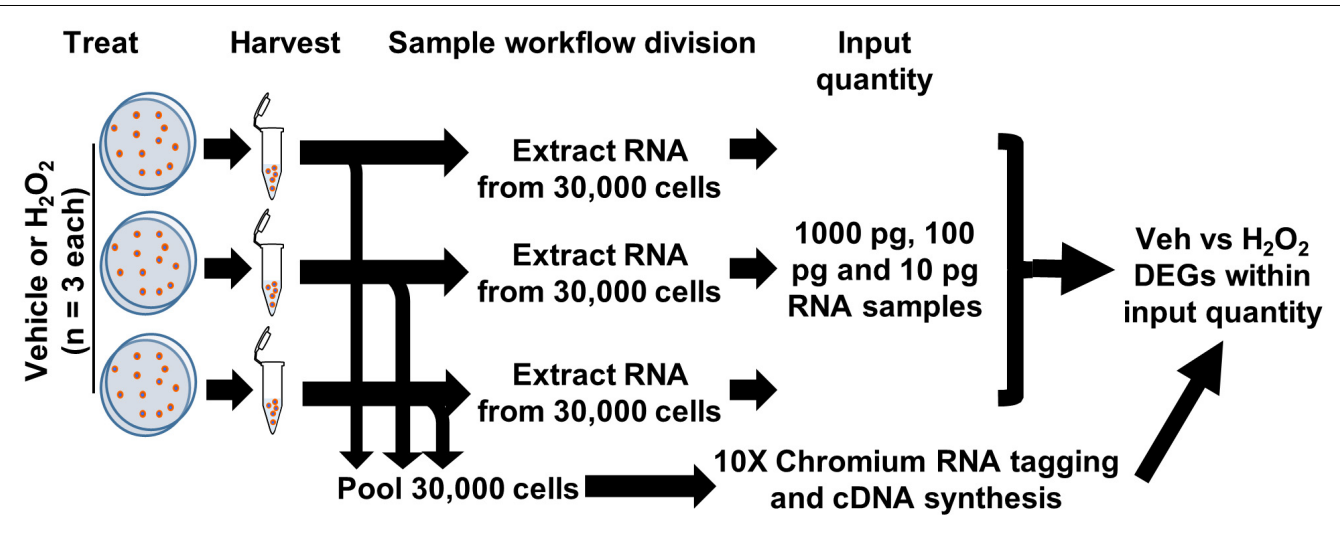

FIGURE 1 | Workflow for cultured NSCs in scRNAseq and bulk RNAseq. NSCs were treated with $\mathrm{H}_{2} \mathrm{O}_{2}$ or vehicle in triplicate. 10,000 cells from each biologic replicate were pooled for a total of 30,000 cells applied to the $10 \times$ Chromium scRNAseq platform and subsequent DEG analysis. RNA was extracted from 30,000 cells of each biologic replicate and 10,100, and 1,000 pg (1 ng) were used in RNAseq with subsequent CLEAR filtering and DEG analysis.

treated cultured NSCs derived from male and female adult mouse DGs with $\mathrm{H}_{2} \mathrm{O}_{2}$ to induce oxidative stress or with vehicle and then harvested cell pellets from three biological replicates (one male and two female) per treatment. Harvested cells were then subdivided into two processing streams: RNA extraction for bulk RNAseq or direct RNAseq of individual cells (scRNAseq) on the $10 \times$ Chromium platform (Figure 1).

For scRNAseq, a total of 26,916 cells were captured and sequenced. UMAP analysis of both $\mathrm{H}_{2} \mathrm{O}_{2}$-treated and control samples revealed 10 different subpopulations characterized by gene expression profiles linked to GO terms consistent with specific stages of the cell cycle, phases of quiescence, differentiation, or response to injury (Figures $\mathbf{2 A}, \mathbf{B}$ and Supplementary Table 1). The majority of cells were in $\mathrm{G}_{1}(22 \%)$, $\mathrm{G}_{2} / \mathrm{M}(21 \%)$, or $\mathrm{S}(6.5 \%)$ phase of the cell cycle. $17.5 \%$ of cells were in one of two detected quiescent phases $\left(\mathrm{G}_{0 \mathrm{~d}}\right.$ and $\left.\mathrm{G}_{0 \mathrm{r}}\right)$. $16 \%$ of the cells appeared to be in an intermediate state that we characterize as transitioning to/from the cell cycle $(\mathrm{T})$ and the remaining $17 \%$ of cells were differentiating $\left(3 \%, \mathrm{D}_{1}\right.$ and $\left.\mathrm{D}_{2}\right)$ or responding to injury (14\%, I and A). Cells from both treatment groups were present in all clusters (Figure 2A). However, vehicle treated cells were mostly concentrated in the cycling clusters while $\mathrm{H}_{2} \mathrm{O}_{2}$ treatment resulted in a notable shift away from cycling clusters to quiescent and apoptotic/injured clusters.

To confirm the cluster identities of the dormant and cycling cells, we looked at expression of known markers of dormancy and progression through the cell cycle. The largest subpopulation, which we designated as $\mathrm{G}_{1}$ based on $\mathrm{GO}$ analysis, was characterized by moderate to high expression of genes involved in exit from dormancy, such as Rpl5, Ran, and Rps17 (Harris et al., 2021), as well as moderate expression of cell cycle genes such as Top2a, Cdk1, and Mki67 (Figures 2A,B) (Diril et al., 2012; Sun and Kaufman, 2018; Nielsen et al., 2020). The $\mathrm{S}$ phase population was characterized by a distinct upregulation of cell cycle gene expression (Figure 2B). The $\mathrm{G}_{2} / \mathrm{M}$ cluster showed continued high expression of exit from dormancy genes coupled with sharp downregulation of cell cycle genes relative to $G_{1}$ and $S$ (Figures 2A,B). Two clusters of $G_{0}$-like cells were found, both of which showed upregulation of genes linked with GO terms such as ion homeostasis and metabolic processes. The two clusters differed most notably in expression of genes associated with transition to/from deeper quiescence. Specifically, $\mathrm{G}_{0}$-dormant was characterized by high expression of quiescenceassociated genes Fabp7, Aldoc, Sparc, Clu, and Id3 (Artegiani et al., 2017; Dulken et al., 2017; Urbán et al., 2019; Borrett et al., 2020), coupled with low expression of exit from dormancyassociated genes Rpl5, Rps17, and Ran (Figures 2A,B) (Harris et al., 2021). $\mathrm{G}_{0}$-rest, in contrast, showed an upregulation of exit from dormancy genes and slight suppression of quiescence genes (Figures 2A,B). The transition group (T) was intermediate in expression of markers of dormancy and cell cycle, supporting its assignment as a transitional state between quiescent $\mathrm{G}_{0}$ states and the cell cycle (Figures 2A,B). The two clusters of differentiating cells $\left(D_{1}\right.$ and $\left.D_{2}\right)$ showed upregulation of genes associated with progenitor cell differentiation (Sox4, Sox11, and Map2) (Figures 2A,B) (Shin et al., 2015; Artegiani et al., 2017; Hochgerner et al., 2018). Last, both injured and apoptotic clusters showed expression of genes associated with cell injury and death processes such as Srxn1 and Phlda3 respectively (Supplementary Table 1) (Kawase et al., 2009; Bell et al., 2015). Representative feature plots of two genes significantly upregulated in each cluster are presented in Supplementary Figures 1C-L. Two different methods of cell cycle regression analysis was also performed to remove potential bias from cell cycle-related genes on cell clustering (Supplementary Figures 1A,B). These analyses both yielded similar subpopulations that were still predominantly defined by cell cycle state.

We next identified DEGs between $\mathrm{H}_{2} \mathrm{O}_{2}$ and vehicle treated cells using both the $10 \times$ scRNAseq data and RNAseq from pooled cells using $1 \mathrm{ng}$ total input RNA, an amount which yields sequencing data in the bulk RNA sequencing range (Walker et al., 2020). For scRNAseq, all clusters were combined within treatment for DEG analysis using the standard Wilcoxon test. 299 DEGs were identified between $\mathrm{H}_{2} \mathrm{O}_{2}$ and vehicle treated NSCs using scRNAseq (Supplementary Figures 2A,B and Supplementary Table 2). $1 \mathrm{ng}$ RNAseq data was pre-processed 


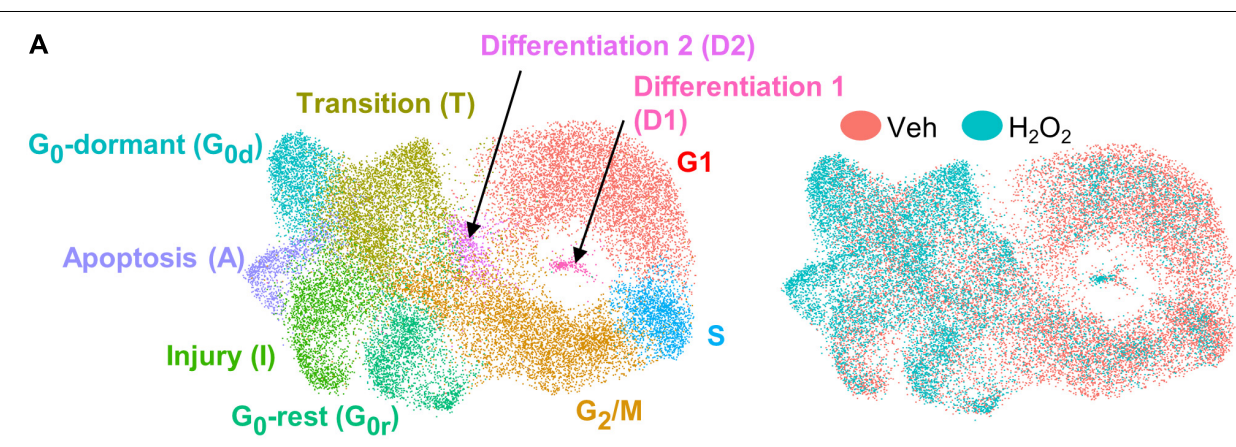

B

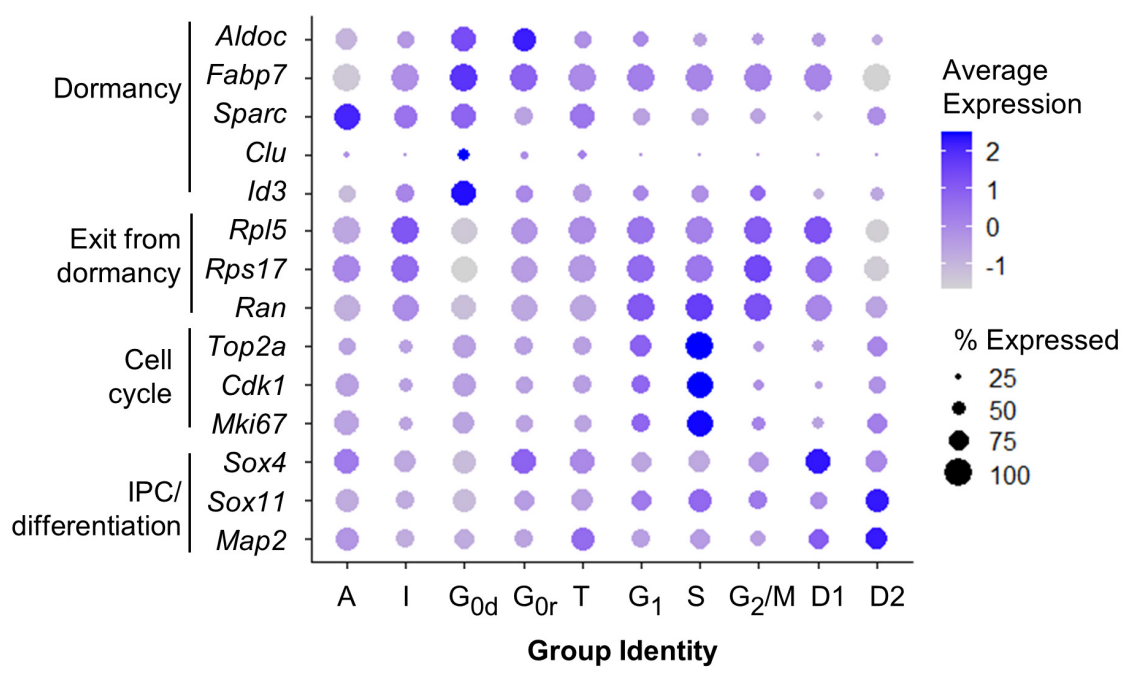

FIGURE 2 | scRNAseq of cultured NSCs in an in vitro model of oxidative stress. (A) UMAP of vehicle and $\mathrm{H}_{2} \mathrm{O}_{2}$-treated cultured NSCs yielded 10 subpopulations defined by gene expression profiles consistent with GO terms associated with various stages of the cell cycle, levels of quiescence or response to injury (left). UMAP comparison of $\mathrm{H}_{2} \mathrm{O}_{2}$-treated versus vehicle-treated NSCs indicated a shift toward quiescence, apoptosis, senescence, and injury response following oxidative stress (right). (B) Dot plot visualization of average expression of and percent of cells expressing select genes known to be associated with quiescence versus activation further confirmed subpopulation identities.

using the coverage-based limiting-cell experiment analysis (CLEAR) pipeline to eliminated unreliable, lowly expressed transcripts (Walker et al., 2020) then DEGs were identified using DESeq2. The 1ng RNAseq comparison yielded 790 DEGs between $\mathrm{H}_{2} \mathrm{O}_{2}$ and vehicle treated NSCs (Supplementary Figures 2A,B, and Supplementary Table 2). Comparison with the DEGs identified between sequencing methods revealed only 93 genes that were common to both platforms (Figure 3A). Not surprisingly, most of the non-overlapping genes (697) were unique to the higher RNA input platform of $1 \mathrm{ng}$ RNA, implying an expected greater sensitivity for DEG detection with greater RNA input. More unexpectedly, though, scRNAseq identified 206 unique DEGs compared to the $1 \mathrm{ng}$ input RNAseq. This low overlap between sequencing approaches from the same source NSCs implied either that one method was calling numerous false DEGs or that the two platforms had strongly different biases in what DEGs they can detect.

To determine how accurate and replicable the DEGs from $10 \times$ scRNAseq and $1 \mathrm{ng}$ RNAseq were, we repeated $\mathrm{H}_{2} \mathrm{O}_{2}$ /vehicle treatment of cultured NSCs in two more independent replications and analyzed gene expression of the top upregulated DEGs identified by scRNAseq and $1 \mathrm{ng}$ RNAseq using quantitative real time PCR (qRT-PCR). Of the top 20 DEGs, we achieved effective primers for 19 of the scRNAseq DEGs and 16 of the $1 \mathrm{ng}$ RNAseq DEGs. The majority of genes identified by both scRNAseq and 1 ng RNAseq were confirmed to be upregulated with qRT-PCR and there was no significant difference between methods in the number of genes replicated (Figure 3B and Supplementary Figure 2C). To further understand what distinguished DEGs called by scRNAseq versus 1 ng RNASeq, we compared overlap of GO terms for upregulated genes in each platform. GO terms showed a high degree of overlap (35 out of 45 distinct categories) in the biological processes represented by the genes identified with both scRNAseq and 1 ng RNAseq (Figure 3C and Supplementary Table 2). This overlap in biological processes being triggered by oxidative stress, coupled with the high replicability of individual DEGs, implies that both platforms likely generated accurate DEGs that reflect true changes in cell activity. However, the low degree of overlap in the individual DEGs identified by the 2 platforms indicates that some form of bias affected the type of genes identified by each platform. 

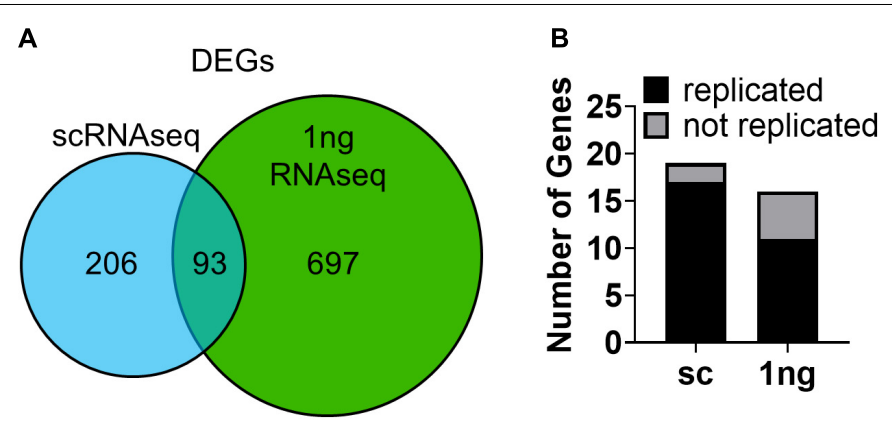

C

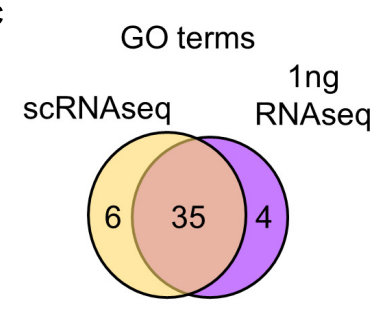

D
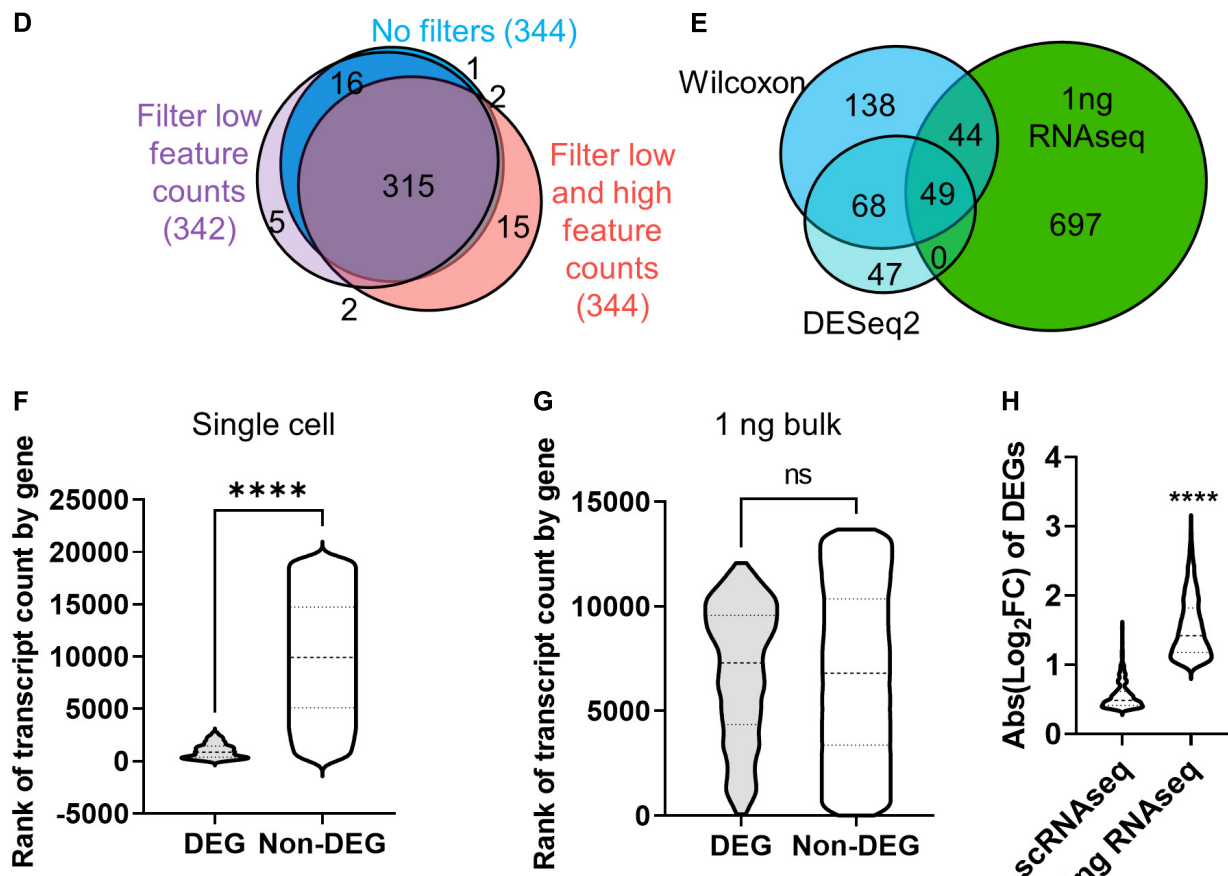

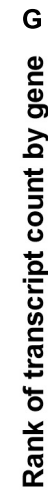

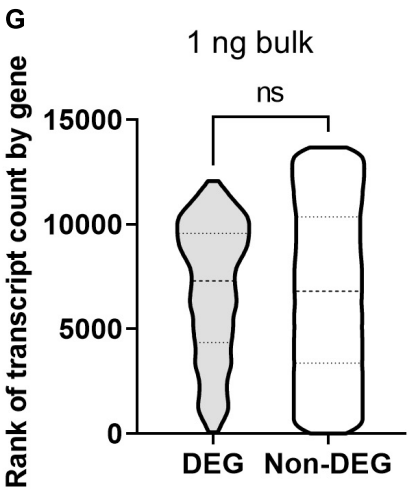

FIGURE 3 | RNA input leads to bias in DEG discovery. (A) Venn diagram of DEGs identified by scRNAseq and 1 ng RNAseq with CLEAR filtering after $\mathrm{H}_{2} \mathrm{O}_{2}$ vs.

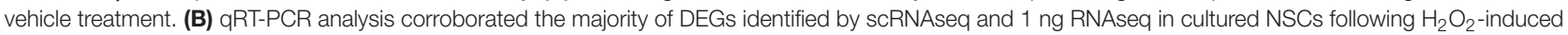
oxidative stress. $X^{2}$ contingency test ( $\left.\mathrm{df}=1\right)=2.331, p=0.127$. (C) Venn diagram of GO terms associated with DEGs identifies by scRNAseq and $1 \mathrm{ng}$ RNAseq. (D) Venn diagram of DEGs identified by scRNAseq with no filtering, filtering for low feature counts, and filtering for both low and high feature counts (i.e., the default for Seurat analysis). (E) Venn diagram of DEGs identified by scRNAseq using the Wilcoxon test or DESeq2 compared with DEGs identified by $1 \mathrm{ng}$ RNAseq. (F,G) DEGs were ranked by average transcript count level relative to all detected gene counts. (F) Violin plot of genes ranked by transcript count level in scRNAseq dataset. DEGs ranked significantly higher in transcript count compared to non-DEGs. ${ }^{* * * *} p<0.0001$ unpaired $t$-test. (G) Violin plot of genes ranked by transcript count level in $1 \mathrm{ng}$ RNAseq dataset. There was no significant difference in rank of transcript count between DEGs and non-DEGs. (H) Comparison of fold changes in average transcript count between treatment groups for DEGs from sc- and $1 \mathrm{ng}$ - RNAseq indicated that DEGs identified by 1 ng RNAseq showed significantly larger fold change in gene expression than DEGs identified by scRNAseq.

One recent study similarly found divergence in DEG detection between single cell and bulk approaches but concluded that this represented error on the part of the single cell data (Squair et al., 2021). A core piece of evidence leading to this conclusion was a high rate of false positive DEGs generated from single cell data when treatment versus control conditions were assigned randomly. This finding is in stark contrast to the findings of Soneson and Robinson (2018), which showed that with the Wilcoxon test now used in Seurat analysis, false positives are not above expected levels (Soneson and Robinson, 2018). To further probe our own single cell data for propensity toward false positive signal, we randomly assigned cells to two groups and performed DEG analysis as done for the actual treated experimental design. With standard limits, no genes met the log fold change threshold ( $\log _{2} \mathrm{FC}$ range: 0.044 to -0.052 ) therefore no DEGs were detected. Our findings therefore align more so with Soneson and Robinson (2018) and do not suggest excessive false positives from single cell data.

\section{RNA Input Amount Drives Bias in Differentially Expressed Genes Discovery}

The first possibility we considered for the difference in DEG identification between sequencing platforms was that it was an 
artifact of the different data analysis and statistical techniques used to identify DEGs in $10 \times$ scRNAseq versus $1 \mathrm{ng}$ RNAseq data. scRNAseq analysis involves several layers of filtering to compensate for technical limitations of low capture efficiency, high dropouts, and doublets. Two commonly used quality controls (QCs) set minimum and maximum thresholds for Unique Molecular Identifiers (UMIs) and feature counts to filter out damaged cells or doublets (Stuart et al., 2019). However, such filtering relies on predetermined values and could overzealously filter out valid cells on the ends of the spectrum of true cellular differences in RNA content and complexity. To determine if QC limits altered DEG discovery, DEG analysis was performed on scRNAseq data with and without filtering for low (1,000 counts/cell) and high (2,500 counts/cell) feature counts (Figure 3D). Removing both high and low feature counts resulted in a total of 344 DEGs with $92 \%$ overlap compared to fully filtered data (Figure 3D). Filtering out only cells with low feature counts yielded 342 DEGs with almost $93 \%$ of those genes also identified when all QCs are employed (Figure 3D). This comparison revealed high overlap in the DEGs identified with and without the various filters, indicating that QC filtering of scRNAseq data was not introducing the divergence in DEG discovery between sequencing approaches.

We next considered whether the different statistical tests used in scRNAseq and $1 \mathrm{ng}$ RNAseq might have contributed to differential DEG identification. For $10 \times$ scRNAseq data, we used the default in Seurat pipeline implementation of the Wilcoxon test. Bulk RNAseq, in contrast, is typically (and was in our case) analyzed using DESeq2. To determine if the choice of statistical test affected the DEGs identified, we compared DEGs called by DESeq2 in both scRNAseq and 1ng RNAseq. DEG discovery using DESeq2 of scRNAseq data resulted in far fewer genes (a little over half the number identified using the Wilcoxon test), but $71 \%$ of those genes were common to both statistical methods (Figure 3E). DESeq2 has been noted to be more restrictive (Mou et al., 2020) so the fewer DEGs from that analysis is not surprising. While the two different methods of statistical analysis of scRNAseq data yielded highly overlapping sets of genes, they both only marginally overlapped (about 30\%) with DEGs identified with DESeq2 in the $1 \mathrm{ng}$ bulk RNAseq dataset (Figure 3E). This comparison suggests that use of Wilcoxon versus DESeq tests is not likely the source of DEG discordance between scRNAseq and 1 ng RNAseq approaches. Rather, these data suggest that there is something inherently different in the data generated with scRNAseq versus $1 \mathrm{ng}$ RNAseq that is leading to different biases in DEG discovery with each approach.

To further explore the differences in DEGs identified in $10 \times$ scRNASeq versus $1 \mathrm{ng}$ RNAseq, we next looked at the relative transcript count level of DEGs. Gene expression level within a cell impacts the likelihood of transcript capture and is known to significantly influence DEG analysis in single cell studies (Mou et al., 2020). Indeed, the low RNA input of scRNAseq is expected to result in high drop out and a consequent overall reduction of detected genes and therefore DEGs. The identification of DEGs in scRNAseq that were not identified via $1 \mathrm{ng}$ RNAseq in our data, however, is more unexpected. To determine if gene expression level led to the discordance in DEG discovery here, the averaged transcript counts per cell of DEGs and non-DEGs identified in scRNAseq and 1ng RNAseq were compared (Figures 3F,G). First, genes that were not expressed in any cells were excluded to limit bias from zero inflation, especially in scRNAseq. DEGs identified by scRNAseq derived from genes with distinctly high transcript counts and spanned a much narrower range of transcript count ranks than non-DEGs (Figure 3F). Meanwhile, a similar comparison of DEGs and non-DEGs from the 1ng RNAseq dataset revealed no significant difference in rank of transcript counts (Figure 3G). It is important to note that these comparisons were made after eliminating genes with no counts, showing that a strong bias for high count genes persists in scRNAseq data even when undetected genes are excluded.

We next analyzed the relative fold change in counts of DEGs identified by $10 \times$ scRNAseq and $1 \mathrm{ng}$ RNAseq. We found that scRNAseq yielded DEGs with lower fold changes than $1 \mathrm{ng}$ RNAseq did. scRNAseq dataset DEGs showed an average fold change less than 2 (Figure $\mathbf{3 H}$ ). Our $1 \mathrm{ng}$ RNAseq data, in contrast, had a threefold change on average. This is, of course, partly driven by the different default thresholds for $\log _{2} \mathrm{FC}$ in these two analysis streams, with DESeq2 using a minimum of 1 and Wilcoxon using 0.36. However, if the scRNAseq data were restricted to a $\log _{2} \mathrm{FC}$ cutoff of 1 , it would only yield 8 DEGs, rather than 299. Volcano plots further emphasize the restricted nature of $\log _{2} \mathrm{FC}$ in the $10 \times$ scRNAseq data compared to $1 \mathrm{ng}$ bulk RNAseq (Supplementary Figure 3B). Combined with the above findings on relative count level of DEGs, these findings suggest that the divergence in DEG detection between our scRNAseq and $1 \mathrm{ng}$ RNAseq data is driven by bias in scRNAseq data for detection of DEGs that derive from high count genes that show a more moderate fold change and in $1 \mathrm{ng}$ RNAseq data for DEGs from more moderate count genes that show a higher fold change between groups.

\section{RNAseq Determination of Optimal RNA Input for Differentially Expressed Genes Discovery}

The comparison of $10 \times$ scRNAseq and $1 \mathrm{ng}$ RNAseq shows that while both methods accurately identify DEGs, RNA input biased the types of DEGs that were detected. In studies seeking to identify specific causative genes implicated in a manipulation (e.g., disease model, drug treatment, and altered gene expression), discovering genes that have high fold change and low expression level is particularly advantageous. Our data suggest that scRNAseq may be distinctly ill-suited for this purpose. For many applications, a bulk RNAseq approach may therefore be desirable. However, in the case of in vivo NSCs, the number of cells isolated from a single mouse is lower (100s1,000 s) than that which is typically necessary for bulk RNAseq $(100,000 \mathrm{~s})$. We used our in vitro oxidative stress model to determine the consequences of reduced RNA input, like that which may be encountered in a limiting cell (lc) RNAseq analysis of in vivo NSCs, for DEG detection. From the same samples, we compared transcriptional profiles derived from $1 \mathrm{ng}, 100 \mathrm{pg}$, and $10 \mathrm{pg}$ of RNA input. Similar to our analysis of the 1 ng samples 
above, 100 and 10 pg RNAseq data was pre-processed using the CLEAR pipeline to eliminate unreliable, lowly expressed transcripts (Walker et al., 2020). Principal component analysis (PCA) showed a clear separation between $\mathrm{H}_{2} \mathrm{O}_{2}$ - and vehicletreated NSCs on principal component (PC) 1 (Figure 4A). The clearest separation, however, was by RNA input level, along PC2 (Figure 4B).

Comparison of the median coefficient of variation (CV) between biological replicates showed that $\mathrm{CV}$ increased significantly with decreasing RNA input (Figure 4C). However, the difference in median CV was much more moderate between $1 \mathrm{ng}$ and $100 \mathrm{pg}$ ( $46 \%$ increase from $1 \mathrm{ng}$ to $100 \mathrm{pg}$ ) versus $1 \mathrm{ng}$ and $10 \mathrm{pg}$ ( $\sim 77 \%$ increase). These data indicate lower precision and reproducibility as RNA input is reduced, particularly when it drops below 100 pg (Figure 4C). Notably, CV was substantially larger in all conditions when data were analyzed without CLEAR pre-processing, emphasizing the utility of this step for extraction of reliable data in RNAseq analysis (Figure 4D). Comparison of DEGs identified in the CLEAR-processed $10 \mathrm{pg}, 100 \mathrm{pg}$, and $1 \mathrm{ng}$ RNAseq datasets revealed $41.5 \%$ of DEGs overlapped between the $100 \mathrm{pg}$ and $1 \mathrm{ng}$ datasets, while only $9.0 \%$ of the $10 \mathrm{pg}$ and $1 \mathrm{ng}$ DEGs overlapped. $100 \mathrm{pg}$ and $10 \mathrm{pg}$ shared only $10.6 \%$ of DEGs (Figure 4E). Analysis of gene transcript counts for DEGs versus non-DEGs at these RNA input levels revealed that the 100 pg RNA input DEGs had a median transcript count level comparable to that of non-DEGs and, more importantly, this level was similar to that of the $1 \mathrm{ng}$ input (Figure 4F). On the other hand, the 10 pg RNA input dataset exhibited a significantly lower transcript count rank of DEGs, indicating that these DEGs consisted of genes that were more highly expressed (Figure 4F). Cumulatively, these data indicate that using $100 \mathrm{pg}$ of input RNA preserves data quality and many DEG characteristics of sequencing at the $1 \mathrm{ng}+$ level without requiring its substantially higher number of cells. 10 pg of RNA, in contrast, shows greater variability across biological replicates and has less breadth in the count level of detected DEGs.

\section{Limiting Cell RNAseq Enables Differentially Expressed Genes Discovery From Fluorescence Activated Cell Sorting Isolated Neural Stem Cells and Intermediate Progenitor Cells From Individual Mouse Hippocampi}

Standard bulk sequencing of adult hippocampal NSCs, a particularly sparse in vivo population, requires pooling of several mice to generate sufficient quantities of input RNA. Our findings suggest that when using CLEAR pre-processing, RNA input can be decreased substantially in a limiting cell RNAseq (lcRNAseq) approach and still yield reliable DEGs from a wide range of gene count levels. To test whether an RNAseq approach would be useful for transcriptional sequencing of in vivo NSCs, we used FACS to isolate NSCs and IPCs from 3 individual Nestin-GFP transgenic mice (Mignone et al., 2004) (Figure 5A). In these mice, NSCs and IPCs express GFP driven by regulatory elements of the Nestin gene. Using immunofluorescence of fixed tissue sections of adult Nestin-GFP mice, we confirmed that GFAP + SOX2+ radial glia like (RGL) NSCs and SOX2+ IPCs, but not DCX+ neuroblasts/immature neurons or NEUN+ mature neurons, expressed Nestin-GFP (Supplementary Figures 3A-D). We also confirmed Nestin-GFP expression in CD31+endothelia and OLIG2+oligodendroglial cells, as expected based on previous work (Supplementary Figures 3E,F) (Artegiani et al., 2017). To exclude the endothelia and oligodendroglial cells, we selected for cells immunonegative for CD31, O1, and $\mathrm{O} 4$. To specifically separate NSCs from IPCs, we used GLAST immunolabeling, which is a common marker for distinguishing NSCs (LlorensBobadilla et al., 2015). In this design, Nestin-GFP + GLAST+ cells represent NSCs and Nestin-GFP + GLAST - cells represent IPCs. To maximize RNA integrity, cells were sorted directly into lysis buffer and converted to cDNA libraries without an intervening RNA isolation step. Direct cDNA synthesis prevented measurement of RNA yield to compare with our in vitro studies, but we used the equivalent of a 60 cell RNA input amount (estimated to approximate 100-200 pg input) to generate three technical replicates for each biological (mouse) replicate with a 300 cell complexity level for RNAseq and CLEAR filtering (Figure 5A). For comparison, we also sequenced in parallel $1 \mathrm{ng}$ of RNA isolated from whole DG of three separate mice.

PCA of NSCs, IPCs, and whole DG revealed that CLEAR pre-processing both decreased the percent variance within NSCs and IPCs compared to PCA performed on raw data, and also separated NSCs from IPCs into non-overlapping populations (Figure 5B). Unfiltered (RAW) CVs for NSCs and IPCs across biological replicates were also more than double the whole DG CV (Figure 5C). CLEAR filtering reduced CVs by over 25\% for NSCs and IPCs. CLEAR filtering also reduced the CV between technical replicates within the NSCs (by 25\%) and IPCs (by 28\%) (Figure 5D). Because tissue processing and handling can introduce variability when assessing freshly isolated cells, it was not surprising that the technical and biological CVs of NSCs and IPCs here were slightly higher than that obtained with $100 \mathrm{pg}$ RNA input in vitro. However, the significant improvement in CVs after CLEAR application confirm its utility in improving transcriptional data from limited starting material.

FACS-isolated NSC and IPC population identities were confirmed with expression of characteristic cell type markers for NSCs and IPCs (Figure 5E) (Yuzwa et al., 2017; Hochgerner et al., 2018). Higher relative expression of quiescent radial glial-like cell (qRGL) markers $\beta 2 m, I d 1, I d 3$, and Vim by the NSC samples over the whole DG was observed compared to the relative expression by IPC samples, confirming accurate FACS isolation of NSCs (Figure 5E). Likewise, higher relative expression of IPC markers such as Calb2, Neurog2, Stmn2, and Tubb3 in the IPC samples over the whole DG samples were observed compared to the NSC samples, confirming accurate FACS isolation of IPCs (Figure 5E). When compared to another published scRNAseq dataset including NSCs and IPCs (Hochgerner et al., 2018), we found similar enrichment for common NSC/IPC phenotypic markers in our isolated populations as generated by single cells that were assigned phenotype post hoc based on their individual transcriptomes (Supplementary Figures 4A,B). Exclusion of other cell populations was confirmed via expression 

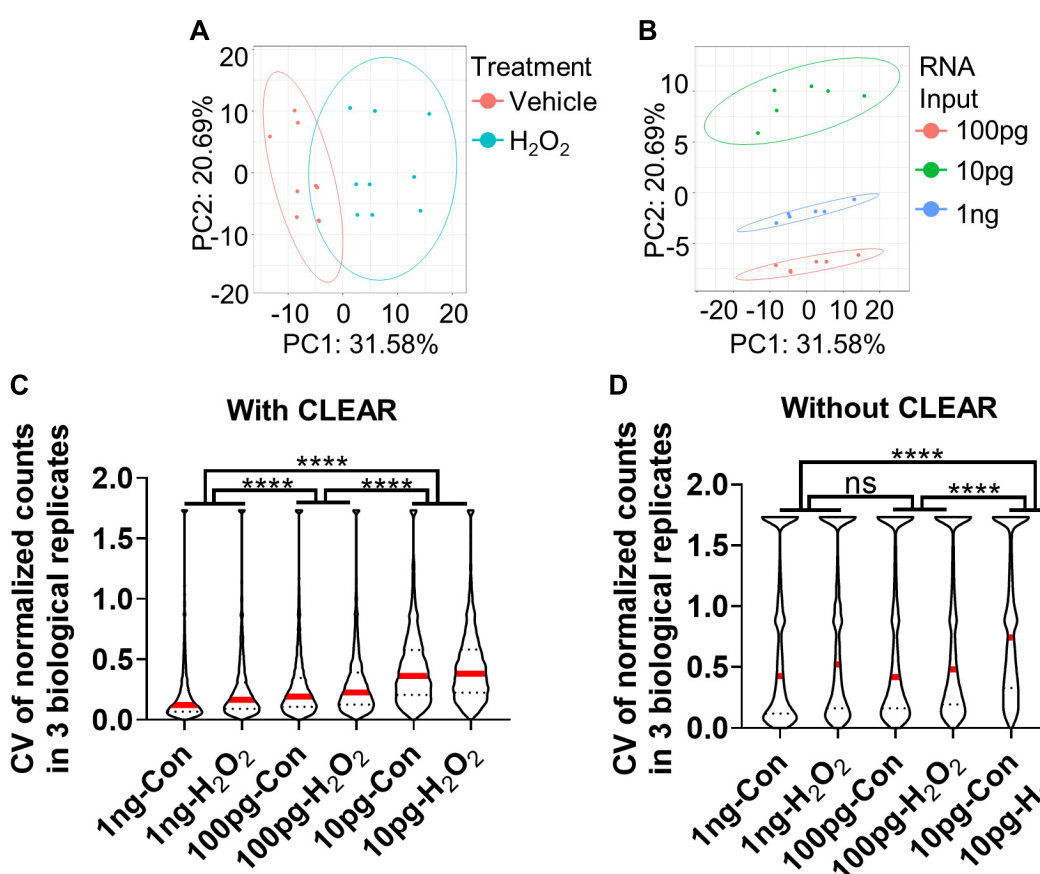

D

$\mathbf{E}$

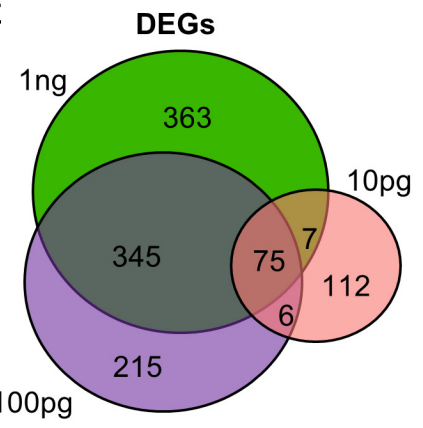

Without CLEAR
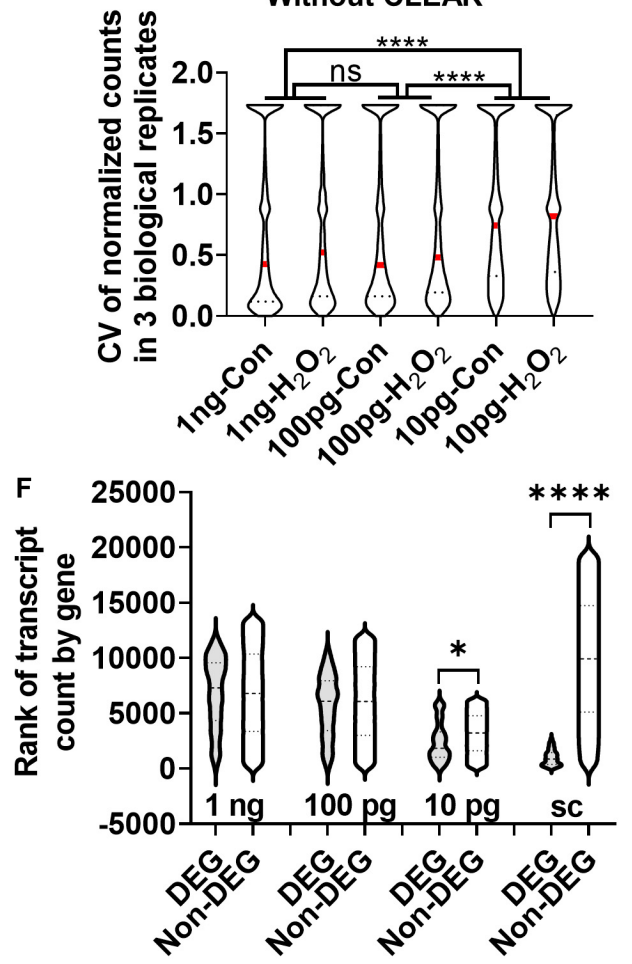

FIGURE 4 | Determination of optimal input RNA amount with RNAseq and CLEAR that preserves unbiased DEG identification. (A) PCA of cultured NSCs shows that cells undergoing oxidative stress diverge from control cells along principal component (PC) 1. (B) PCA of sample based on RNA input amount leads to significant separation between samples along PC2. (C) CVs were inversely related to RNA input amount. The difference in median CV between all RNA input levels were statistically significant. Median (red line) and quartiles (dotted lines) are shown within violin plots of CV for all genes detected in all samples. (D) Analysis of the CV with vehicle and $\mathrm{H}_{2} \mathrm{O}_{2}$-treated samples of all genes detected in all three RNA inputs before CLEAR filtering shows substantially greater CVs at all RNA input levels, confirming the utility of CLEAR preprocessing. Median (red line) and quartiles (dotted line) are shown within violin plots of CV for all genes detected in all samples. (E) Venn diagram of DEGs identified in all three RNA input levels show good overlap between $1 \mathrm{ng}$ and $100 \mathrm{pg}$ but poor overlap with $10 \mathrm{pg}$. (F) Comparison of ranked transcript counts for DEGs and Non-DEGs identified at the three different RNA input levels show no significant difference in transcript count rank between DEGs and non-DEGs at the $1 \mathrm{ng}$ and $100 \mathrm{pg}$ RNA inputs. There are significant differences at the $10 \mathrm{pg}$ and single cell level. ${ }^{*} p<0.5,{ }^{* * * *} p<0.0001$. One-Way ANOVA with Kruskal-Wallis test and Dunn's multiple comparisons in (C,D,F).

of phenotypic genes for neurons, astrocytes, oligodendroglia, endothelia, pericytes, and microglia (Figure 5E). 177 DEGs were identified between NSCs and IPCs acutely isolated from adult mouse DGs (Supplementary Table 3). Violin plots showing transcript count rank for DEGs and detected non-DEGs show that average DEG rank was significantly higher in DEGs than non-DEGs, but this difference was moderate $(30.6 \%$, average rank 1639 DEG v. 2064 non-DEG) and DEGs still had average count ranks spread throughout the ranks of detectable genes (Figure 5F). All together, these findings indicate that RNAseq of adult DG NSCs and IPCs can be achieved from a single mouse per sample replicate with data quality similar to that derived from more bulk-like RNA sequencing.

\section{RNAseq Identifies Differentially Expressed Genes in Neural Stem Cells and Intermediate Progenitor Cells Induced by Lateral Fluid Percussive Brain Injury}

As proof of principle, we applied our RNAseq workflow to a LFPI model of TBI and identified DEGs in adult mouse 


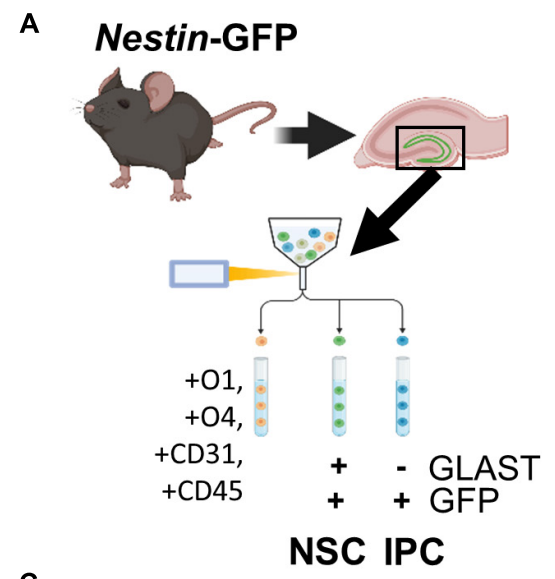

C

E

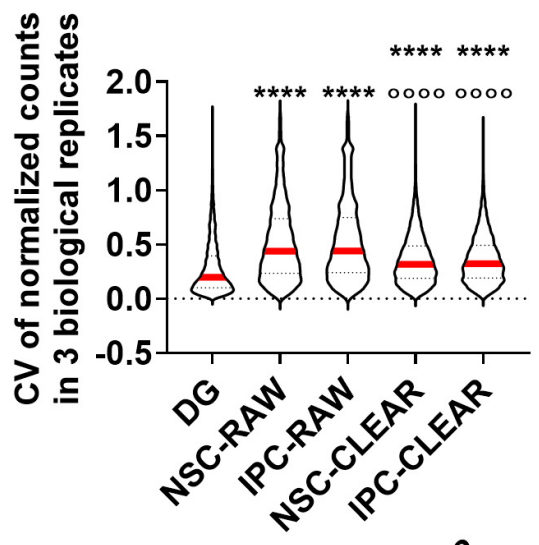

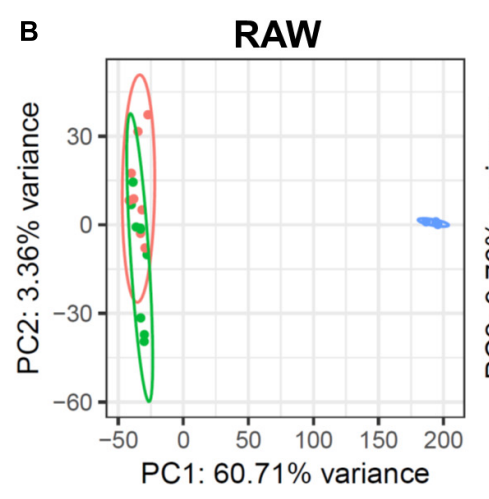
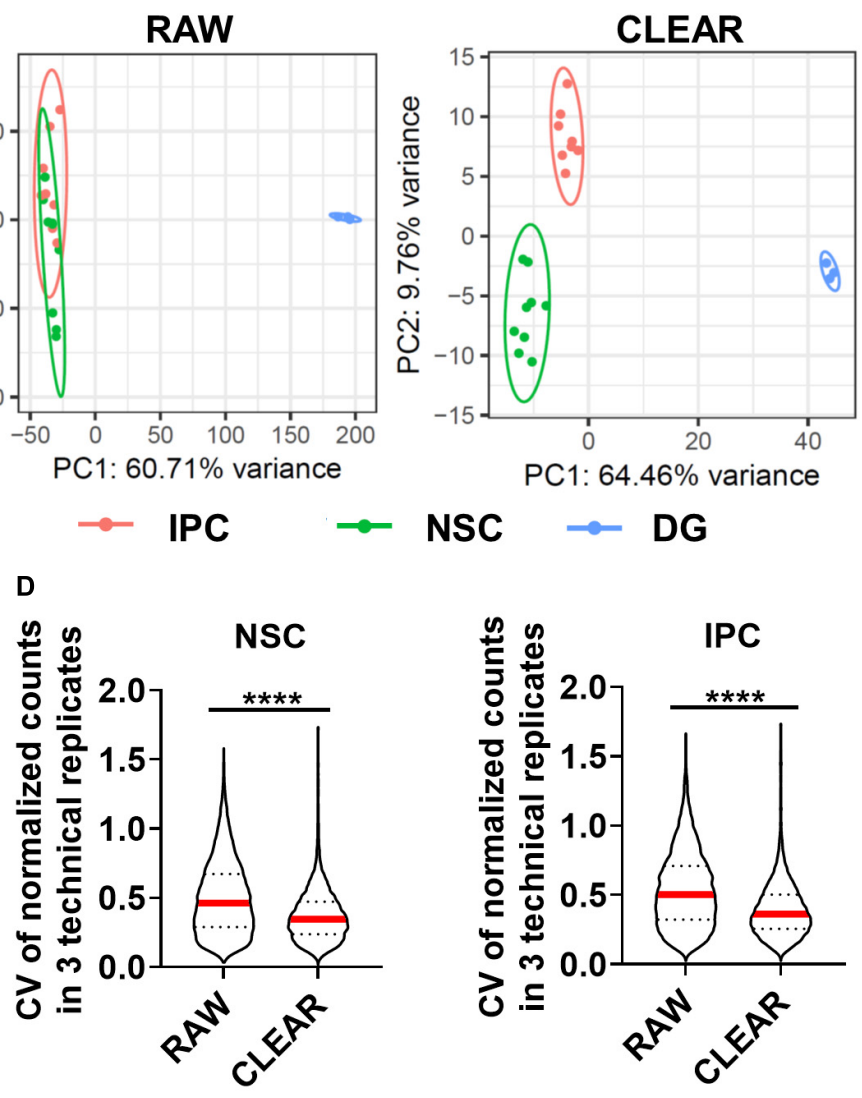

\section{NSC $\rightarrow$ DG}

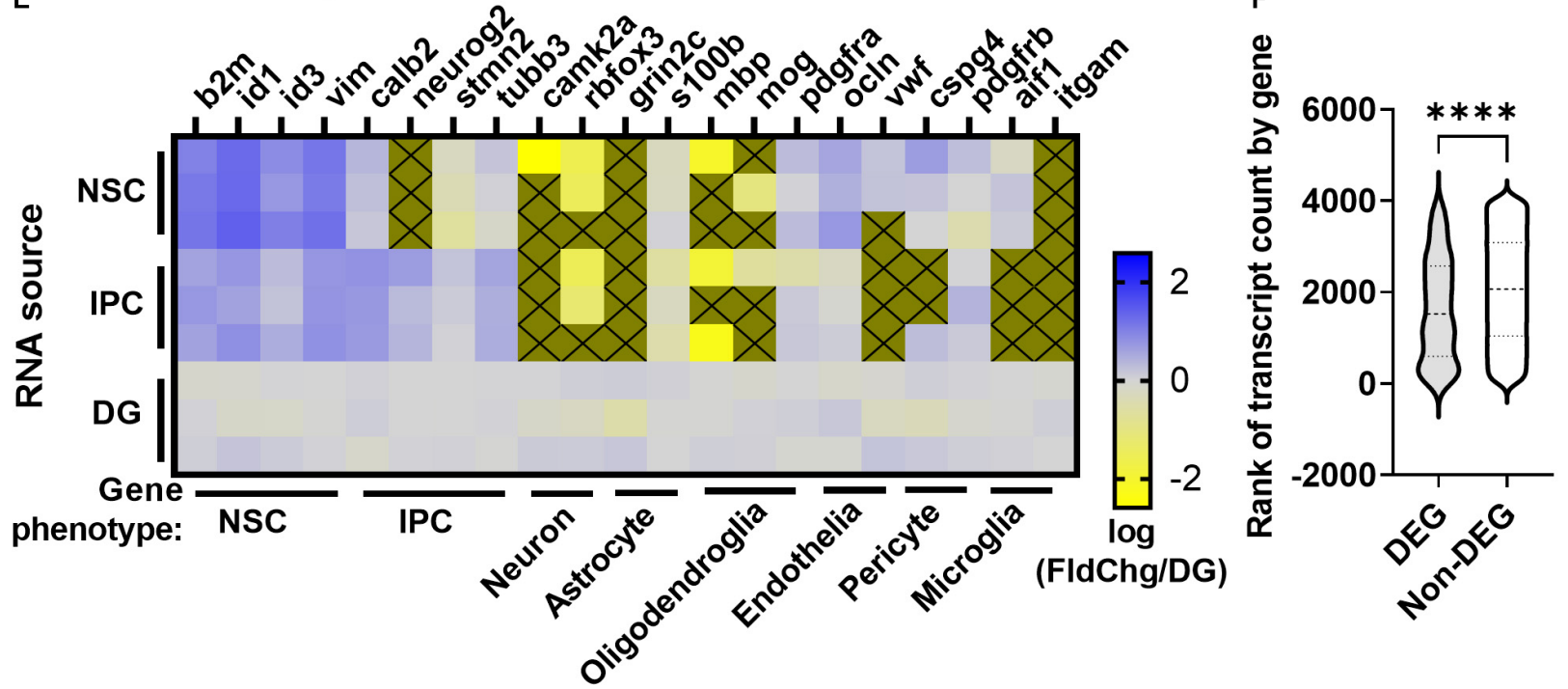

FIGURE 5 | DEG identification in vivo. (A) Workflow for DEG identification from hippocampal NSCs and IPCs of individual adult mice. DGs from Nestin-GFP+ mice were isolated and homogenized into single cell suspensions enabling FACS isolation of NSCs and IPCs for IcRNAseq. (B) PCA of transcriptome using unfiltered RAW and CLEAR filtered data. CLEAR filtering improves separation and variance between NSC and IPC samples. (C) CV for NSC and IPC biological replicates before and after CLEAR filtering. Dunn's multiples comparisons ${ }^{* * * *} p<0.0001$ vs. DG $p<0.0001$ vs. raw RGL and raw IPC. (D) CV for NSC and IPC technical replicates (3/mouse) before and after CLEAR filtering. Unpaired $t$-test ${ }^{* * * *} p<0.0001$. (E) Heatmap shows expression of phenotypic genes in each of three biological replicates for NSCs, IPCs, and whole DG which was sequenced from three separate Wt mice in parallel. High expression of NSC phenotypic markers in NSCs and high expression of IPC phenotypic markers in IPCs confirmed cell type identities. Low expression of neuronal, astrocytic, oligodendroglial, endothelial, pericyte, and microglial phenotypic genes confirmed exclusion of other major DG cell types. Gold boxes with an X were not detected. (F) Comparison of ranked transcript counts for DEGs and Non-DEGs identified between NSCs and IPCs shows a significant but moderate shift in DEGs toward higher ranked genes. Mann-Whitney test ${ }^{* * * *} p<0.0001$. 
NSCs and IPCs in vivo (Figure 6A). NSCs and IPCs from the DGs ipsilateral to injury of three individual mice were FACS isolated $4 \mathrm{~h}$ after LFPI or sham treatment. Whole DGs from separate, but similarly treated, mice were also processed for comparison by $1 \mathrm{ng}$ RNA input sequencing. A total of 6,319 genes were identified in NSCs, IPCs, and whole DGs (Supplementary Table 4). 23 DEGs were identified in NSCs after LFPI with 15 significantly upregulated and 8 significantly downregulated (Figure 6B). In the IPC population, 5 genes were significantly upregulated while 13 genes were downregulated in LFPI mice compared to sham mice (Figure 6C). In whole DG, 188 DEGs were identified with 106 significantly upregulated and 82 significantly downregulated in LFPI mice compared to sham mice (Supplementary Figure 4C). DEGs identified in NSCs and IPCs following LFPI did not overlap with DEGs identified on a whole DG level. In addition, NSCs and IPCs only shared one DEG in common (Slc5a3), emphasizing the importance of examining individual cell types, even for cells as closely related as NSCs and their IPC progeny. DEGs from both NSC and IPCs derived from genes throughout the range of detected genes and showed median count levels similar to nonDEGs (Figure 6D). RNAscope fluorescent in situ hybridization (FISH) combined with immunohistochemical staining verified transcriptional upregulation of $S l c 5 a 3$ in NSCs and IPCs following LFPI (Figures 6E,F). We also verified two other DEGs upregulated in NSCs using RNAscope FISH: Serpina3n (Figure 6G) and Timp1 (Figure 6H). Similar trends were found when individual mouse averages of puncta per cell were used as the replicates ( $n=5$ per group), though not all reached statistical significance (Supplementary Figures 4D-G). These findings indicate that RNAseq of acutely isolated, in vivo NSCs and IPCs can reliably identify DEGs in an injury model.

\section{DISCUSSION}

Endogenous adult hippocampal NSCs provide a source of both cellular and biochemical support for tissue homeostasis. Characterizing these cells at baseline, as well as after injury, may lead to therapeutically relevant strategies for promoting optimal brain function. However, studying adult DG NSCs is challenging due to their relatively low cell number, residence within a complex niche, and their inherent heterogeneity. To overcome this challenge, researchers have increasingly turned to scRNAseq approaches for transcriptional profiling (Shin et al., 2015; Hochgerner et al., 2018; Knight and Serrano, 2018; Zeng et al., 2018; Kulkarni et al., 2019). However, we show here that, despite the many strengths of this approach, scRNAseq may not be the ideal method to answer certain types of research questions. Specifically, we show that scRNAseq analysis of differential gene expression may miss genes that are more moderately expressed and show large fold changes in expression. We show that an lcRNAseq approach can be used to help circumvent this problem and discover DEGs from a broader count range and with greater fold changes, and that such an approach can be adapted for transcriptional profiling and DEG identification in NSCs acutely isolated from adult mouse DG.
Using a model of oxidative stress in cultured NSCs, we identified DEGs from the same source samples using both a $10 \times$ Chromium scRNAseq and 1 ng bulk RNAseq approach. By using cells from the same biological replicates for both sequencing platforms, we avoided potential variation in gene expression induced by a difference in tissue processing. Yet, we found little overlap in the identification of DEGs by these two methods. This lack of overlap was maintained when scRNAseq data were analyzed without filtering for high or low feature counts and when the same statistical method was used for DEG discovery in both datasets, suggesting it was not an artifact of data processing/analysis. Despite this low overlap in DEGs, both scRNAseq and bulk RNAseq yielded accurate DEGs that could be confirmed with qRT-PCR in independent experimental replicates. GO analysis of the DEGs identified using the two different approaches also implicated mostly the same biological pathways being triggered by injury. Furthermore, our own investigation of false positive rate in scRNAseq, when cells were randomly assigned to two groups, also yielded no false DEGs, consistent with findings in Soneson and Robinson (2018), who performed similar analyses over multiple scRNAseq datasets and found low (sometimes no) false discoveries when using the same statistical analysis approach that we used (Wilcoxon). In contrast to these findings, Squair et al. (2021) recently published findings suggesting that divergence of scRNAseq differential expression analysis from that of matched bulk RNAseq represented false positives in scRNAseq. This conclusion was mostly based on simulations where they randomly assigned pseudo-replicates or real replicates to treatment groups. Particularly when replicateto-replicate variation was high, large numbers of false positives were found in this analysis. Squair et al. (2021) also used an RNAscope assay to attempt to confirm scRNAseq-derived DEGs in a model of spinal cord injury and found low replicability there. RNAscope is commonly used to confirm scRNAseq findings so it is unclear why replicability is typically reported in other studies but not found in Squair et al. (2021). Two notable possibilities are: (1) that a bias for reporting positive results exists in previous literature or (2) that the often semi-quantitative nature of RNAscope analysis can mask true differences. Altogether, these findings suggest that the reliability of scRNAseq data requires much more scrutiny than has been applied to-date. Our data adds to that of the meta-analysis in Soneson and Robinson (2018) to suggest low false positive rates when individual cells are used as the input sample for DEG analysis and our data go further to confirm replicability of DEGs identified this way, though those DEGs show strong bias for higher count genes with lower fold changes.

When we probed the difference in the kinds of DEGs identified by scRNAseq versus $1 \mathrm{ng}$ RNAseq, we found that scRNAseq identified high count genes with lower fold changes while $1 \mathrm{ng}$ RNAseq identified genes with a wider range of count levels, including many genes with moderate counts and higher fold changes. Importantly, this bias in count level and fold change emerged in comparison to transcripts detected above 0 in each respective dataset. scRNAseq, of course, yields fewer detected genes than a bulk approach but this difference in DEG profile emerged even among genes that would be recorded in the dataset 
A
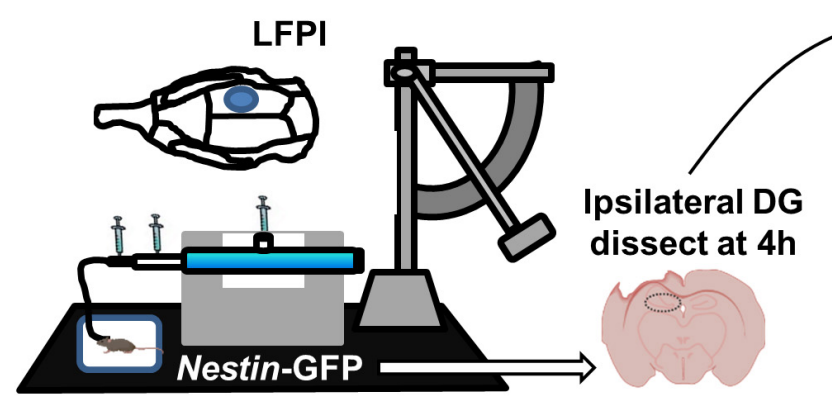

B

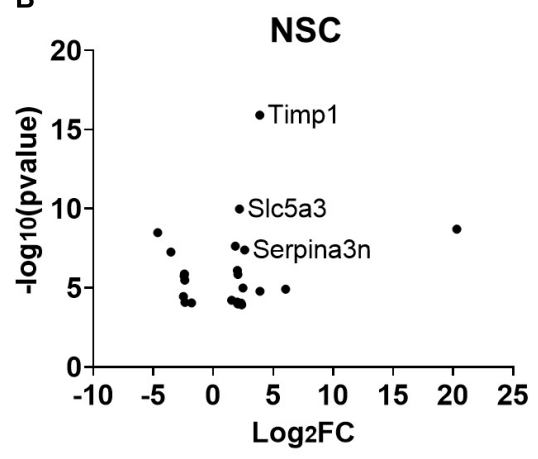

C

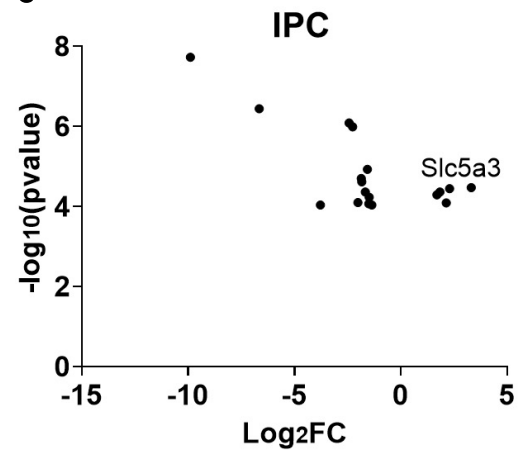

NSC IPC

D

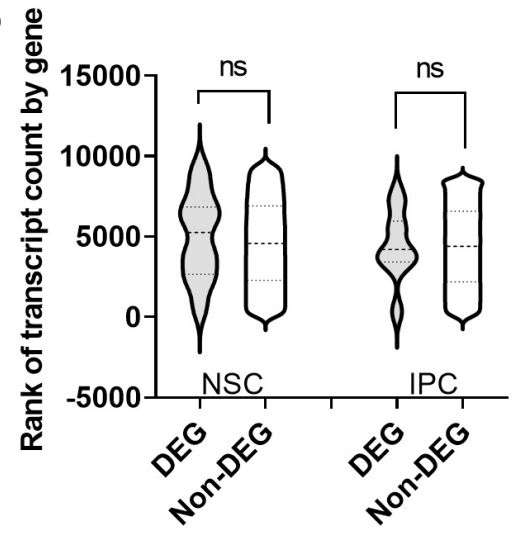

E

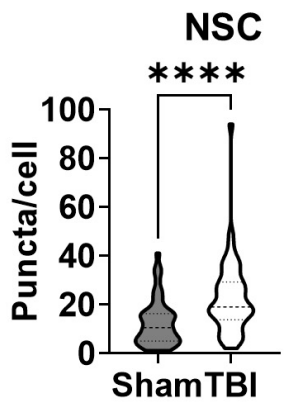

G
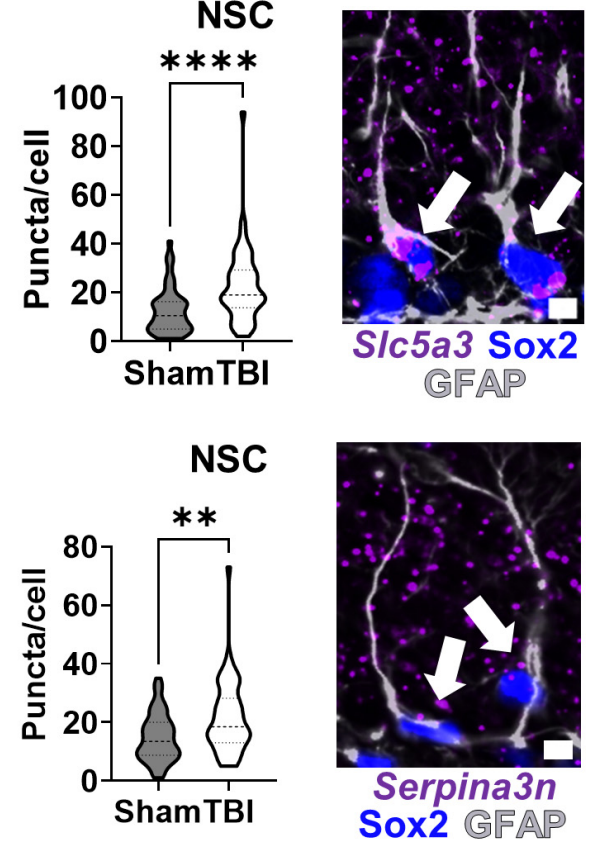

F
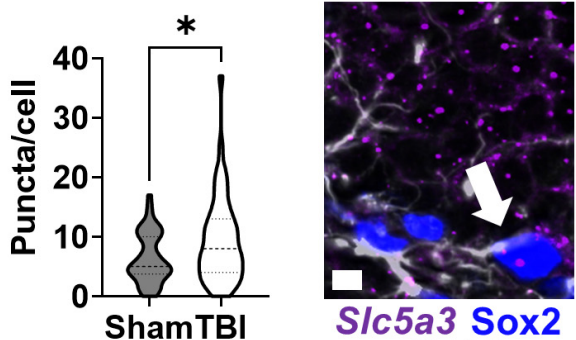

SIc5a3 Sox2

GF迩
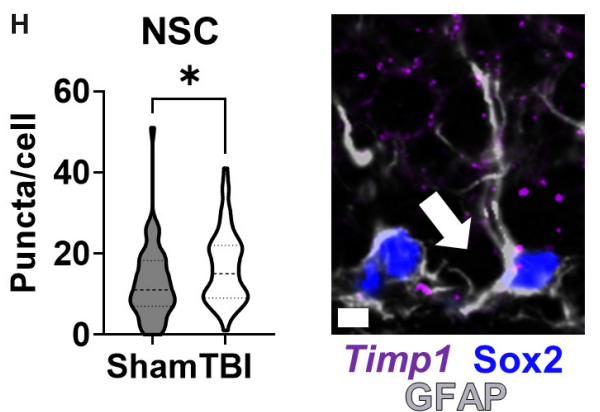

FIGURE 6 | RNAseq and CLEAR filtering enable accurate DEG identification in NSCs and IPCs in mice with lateral fluid percussive brain injury (LFPI). (A) Mice ( $n=3$ per group) were given lateral fluid percussive injuries or sham treatment. Four hours after injury, DGs from the ipsilateral side of the brain were isolated and processed for RNAseq as described in Figure 5A. (B) Volcano plot of DEGs identified in NSCs after LFPI. (C) Volcano plot of DEGs identified in IPCs after LFPI. (D) Violin plot of TBI DEG and non-DEG transcript count level distribution in NSCs and IPCs. ns, not significant, Mann-Whitney test. (E) Quantification of S/c5a3 mRNA via RNAscope in NSCs (identified via GFAP + SOX2+ immunolabeling) confirmed upregulation after TBI (left). Representative image with arrows pointing to NSCs with S/c5a3 expression (right). (F) Quantification of S/c5a3 mRNA in IPCs similarly confirmed increased expression after LFPI (left). Representative image with arrow pointing to IPC with S/c5a3 expression (right). (G) Quantification of Serpina3n mRNA in NSCs also confirmed upregulation after LFPI (left). Representative image with arrow pointing to NSC with Serpina3n expression (right). (H) Quantification of Timp1 mRNA in NSCs also showed increased expression after LFPI (left).

Representative image with arrow pointing to NSC with Timp1 expression (right). (E-H) Mean \pm SEM of mean RNA puncta per cell with $n=50$ cells per group from 5 mice, Mann-Whitney $t$-test, ${ }^{*} p<0.05,{ }^{* *} p<0.01,{ }^{* * * *} p<0.0001$. Scale bars $=5 \mu \mathrm{m}$. 
as detected and therefore having been evaluated for potential to be a DEG. Squair et al. (2021) similarly identified bias in single cell data for high count genes across multiple other datasets, suggesting that this is a common feature of single cell analyses. Squair et al. (2021) also proposed that pseudobulk analysis of single cell samples using biological replicates as the input sample could improve overlap between scRNAseq and bulk RNAseq transcriptomics. Our scRNAseq data was not separated into the original biological replicates so this analysis could not be done in the present study. However, using the data from Squair et al. (2021), the improvement expected by using pseudobulk would be moderate. They presented differential expression overlap between single cell and bulk analysis of parallel samples as an area under the concordance curve metric that can range from 0 to 1 . The best performing pseudobulk analysis method showed only $\sim 15 \%$ median improvement on this scale over Wilcoxon tests performed with individual cells as the input (0.23 Wilcoxon to 0.38 pseudobulk with EdgrR-LRT test). Pseudobulk therefore presents an improvement, but only a marginal one. Taken together, our analyses support the need for additional methods in the transcriptomic toolbox that facilitate inclusion of multiple biological replicates, such as the method we present here.

There are several likely contributors to the selectivity of scRNAseq data for generating DEGs from high count transcripts. The first and most obvious potential contributor is the high zerocount rate in scRNAseq data, due to the very low RNA input from a single cell. Transcripts with counts near threshold of detection will be characterized by many 0 -count genes and therefore have relatively high variability from cell to cell. This high variability may make such genes less likely to be detected as significantly different between two conditions. Discovery of genes with higher counts, and therefore lower zero-influenced variability, would be favored. By being higher count, fold change is similarly likely to be more constrained by a ceiling effect, yielding lower fold change DEGs. Analytical processing compensations for heteroskedasticity may also play a role. Heteroskedasticity is the phenomenon whereby genes with relatively low counts exhibit higher fold changes. Thus, general DEG analysis methods, such as DESeq2, and scRNAseq analysis pipelines, such as Seurat, correct for this problem by applying a variance-stabilizing preprocessing step that transforms the data and minimizes the effect of count-based technical noise on ratio-based outputs such as log fold change in gene expression (Love et al., 2014; Ahlmann-Eltze and Huber, 2021). Although heteroskedasticity is recognized for any count-based data, standard bulk level RNAseq is inherently more robust and delivers generalized expression data for hundreds of thousands of cells. This facilitates accurate correction for heteroskedasticity, while in scRNAseq, low biological replicates and very low input RNA amounts may lead to over-correction of true variation in gene expression by variance-stabilizing preprocessing (Love et al., 2014; Mou et al., 2020).

While our findings suggest that $10 \times$ scRNAseq and lowinput population level RNAseq are both similarly accurate, as measured by replicability, they suggest different utility of each approach depending on experimental goal. If characterization of cellular heterogeneity is the goal, scRNAseq is obviously superior. However, discovery of genes that are moderate to lowly expressed in cells yet exhibit higher fold changes upon stimulation is also a common goal, particularly when searching for candidate molecular mediators or druggable targets in models of injury and disease. Relying on scRNAseq alone for DEG discovery could therefore limit the scope of understanding of disease or injury-associated transcriptional signatures. The difference in DEG characteristics between scRNAseq and 1 ng RNAseq shown here emphasizes the importance of identifying the ultimate goals and readouts of an experiment to choose the method that best addresses the needs of the study.

Although several studies have provided invaluable insight into hippocampal NSC biology using scRNAseq (Shin et al., 2015; Artegiani et al., 2017; Hochgerner et al., 2018), standard bulk RNAseq to study endogenous adult hippocampal NSCs has been challenging, as evidenced by the dearth of such studies. Though NSCs have been successfully profiled when combined with their IPC progeny via bulk RNAseq approach (Adusumilli et al., 2021), NSCs alone are sufficiently rare that their "bulk" level transcriptional profiling is especially difficult. Here, we optimized a protocol that enables population level transcriptional analysis of this rare cell type from individual mice. First, we used cultured adult hippocampal NSCs to determine $100 \mathrm{pg}$ as a lower RNA input amount that enables profiling of DEGs that are comparable to those obtained with standard bulk sequencing. Using this threshold of 100 pg RNA input as a guide for in vivo analysis, we isolated adult DG NSCs and IPCs from individual Nestin-GFP reporter mice to profile the transcriptomes of each population at a 300 cell complexity level.

To ensure accurate identification of DEGs from this low input level, we applied CLEAR filtering which was previously shown to minimize technical noise due to limited RNA input (Walker et al., 2020). In brief, CLEAR preprocessing removes transcripts from analysis that are detected below a threshold. That threshold is determined by observing where on an mRNA transcript sequence RNAseq read fragments map. When reads show preferential mapping to only $3^{\prime}$ and $5^{\prime}$ ends of their mRNA transcripts, it indicates strong RNA degradation, a feature which predominates as transcript count drops. CLEAR filtering removes transcripts with counts below the threshold where most transcripts start to show this pattern of mapping more exclusively toward the $3^{\prime}$ and $5^{\prime}$ ends of their mRNA sequences. CLEAR filtering of our data improved the coefficient of variation between biological replicates for normalized counts at all RNA input amounts in vitro and in vivo, and was essential for effective transcriptional separation of in vivo-isolated NSCs and IPCs via PCA. We showed that this workflow, with CLEAR filtering, accurately profiled the transcriptomes of NSCs and IPCs. This ability to capture population level complexity with a low amount of RNA input is valuable when studying rare cell populations in complex experimental models of disease or injury where maximizing biological replicates is critical but limited by time and labor costs.

To demonstrate the utility of our approach for transcriptional profiling of in vivo NSCs, we applied it in a proof-of-principle experiment identifying TBI-associated DEGs from in vivo NSCs 
and IPCs. Using our workflow, we obtained cell type specific DEGs $4 \mathrm{~h}$ after the LFPI model of TBI. In response to LFPI, NSCs and IPCs showed mostly unique DEGs, with only one exception: both upregulated Slc5a3, a sodium-coupled inositol transporter protein that maintains osmotic pressure in cells and regulates intracellular myo-inositol levels (Andronic et al., 2015). We confirmed transcriptional upregulation of Slc5a3, as well as two other DEGs, Serpina3n and Timp1, with RNAScope in situ hybridization paired with immunofluorescent staining to identify NSCs and IPCs. These findings suggest that our population-level approach to RNAseq of isolated NSCs and IPCs can effectively identify replicable changes in gene transcription after an injury stimulus.

There are several limitations to this study. First, we only compared bulk RNAseq to scRNAseq using a $10 \times$ Chromium platform. It is therefore possible that other approaches to scRNAseq would yield different results than what we found. However, many of the limitations we noted seemed inherent to working with very low RNA input levels and were not exclusive to our experimental platform (in vitro NSCs) or any one approach to data analysis. Second, the specific cell/RNA input levels that we identified as yielding more bulk-like range in DEG count level and fold change may not apply outside of our selected cell population (adult NSCs). RNA content, cellular heterogeneity and capture efficacy of different cell types and tissue sources will likely influence the appropriate cell input needed in other models. Third, transcriptomics does not equate with proteomics. Particularly in NSCs, there appears to be substantial translational priming, in which mRNA is produced but not translated (Denninger et al., 2020; Kjell et al., 2020). Thus, both transcriptional and proteomic analyses are needed to accurately characterize NSCs in health and disease. Lastly, we applied our workflow to the LFPI model of TBI as proof of concept to demonstrate the utility of our method for transcriptional profiling of rare cell types on a population level. While we did identify several DEGs in adult DG NSCs acutely after LFPI, the potential transcriptional changes that occur at later timepoints and in the contralateral DG NSCs are yet unknown and would provide valuable information about how these cells behave in the context of TBI. Future potential applications of our method are not just limited to TBI models. Our method may be applied to any labor-intensive animal model that seeks to interrogate rare cell types, such as certain spinal cord or even hippocampal cell types after spinal cord injury.

\section{CONCLUSION}

We present a comparison of two different approaches to transcriptional profiling, scRNAseq and population level RNAseq, of adult hippocampal NSCs, a rare cell type that is difficult to study in vivo. We found that each had their strengths, as well as weaknesses, which should be balanced with the needs of each specific study. We have shown here that our method for in vivo transcriptional profiling in a bulk-like lcRNAseq approach can provide valuable information about rare cell populations that are traditionally difficult to study in vivo. Thus, we present our workflow as an addition to the transcriptional toolbox for studying limited in vivo cell types moving forward.

\section{DATA AVAILABILITY STATEMENT}

The datasets presented in this study can be found in online repositories. The name of the repository and accessions number can be found below: National Center for Biotechnology Information (NCBI) Gene Expression Omnibus (GEO), https://www.ncbi.nlm.nih.gov/geo/, GSE189572, GSE189573, GSE189574, GSE189575, and GSE138381 (GSM4308545 only).

\section{ETHICS STATEMENT}

The animal study was reviewed and approved by the Institutional Animal Care and Use Committee (IACUC) at the Ohio State University in accordance with institutional and national guidelines.

\section{AUTHOR CONTRIBUTIONS}

JD: execution of research and writing. JD and EK: primary contributors to research design, analysis, and writing. EK: funding acquisition and supervision. LW, XC, AT, AP, RB, and PY: design, execution, analysis, and writing of methods for RNAseq studies. ZT and OK-C: LFPI studies. SS and RR: provide the general support in execution of research. All authors contributed to the article and approved the submitted version.

\section{FUNDING}

This study was supported by R00NS089938 and R01NS124775 to EK from NIH/NINDS, a Chronic Brain Injury Pilot award to EK and OKC, R50 CA211524 to PY from NIH/NCI, P30CA016058 to the OSU Comprehensive Cancer Center Shared Genomics Resource from NIH/NCI, and P30NS045758 to the Center for Brain and Spinal Cord Repair from NIH/NINDS.

\section{ACKNOWLEDGMENTS}

We thank all of the members of the EK lab for excellent discussions during the execution of this project as well as planning and writing of this work. We also thank the OSU Chronic Brain Injury Initiative (CBI), the OSUCCC Genomics Shared Resource, the OK-C lab, and the OSU animal care staff for their support.

\section{SUPPLEMENTARY MATERIAL}

The Supplementary Material for this article can be found online at: https://www.frontiersin.org/articles/10.3389/fnmol.2022. 810722/full\#supplementary-material 


\section{REFERENCES}

Adusumilli, V. S., Walker, T. L., Overall, R. W., Klatt, G. M., Zeidan, S. A., Zocher, S., et al. (2021). ROS Dynamics Delineate Functional States of Hippocampal Neural Stem Cells and Link to Their Activity-Dependent Exit from Quiescence. Cell Stem Cell 28, 300-314.e6. doi: 10.1016/j.stem.2020.10.019

Ahlmann-Eltze, C., and Huber, W. (2021). Transformation and Preprocessing of Single-Cell RNA-Seq Data Bioinformatics [preprint] doi: 10.1101/2021.06.24. 449781

Andronic, J., Shirakashi, R., Pickel, S. U., Westerling, K. M., Klein, T., Holm, T., et al. (2015). Hypotonic Activation of the Myo-Inositol Transporter SLC5A3 in HEK293 Cells Probed by Cell Volumetry. Confocal and Super-Resolution Microscopy. PLoS One 10:e0119990. doi: 10.1371/journal.pone.011 9990

Artegiani, B., Lyubimova, A., Muraro, M., van Es, J. H., van Oudenaarden, A., and Clevers, H. (2017). A Single-Cell RNA Sequencing Study Reveals Cellular and Molecular Dynamics of the Hippocampal Neurogenic Niche. Cell Rep. 21, 3271-3284. doi: 10.1016/j.celrep.2017.11.050

Arzalluz-Luque, Á, Devailly, G., Mantsoki, A., and Joshi, A. (2017). Delineating biological and technical variance in single cell expression data. Int. J. Biochem. Cell Biol. 90, 161-166. doi: 10.1016/j.biocel.2017.07.006

Babu, H., Claasen, J.-H., Kannan, S., Runker, A. E., Palmer, T., and Kempermann, G. (2011). A protocol for isolation and enriched monolayer cultivation of neural precursor cells from mouse dentate gyrus. Front. Neurosci. 5:89. doi: 10.3389/ fnins.2011.00089

Baser, A., Skabkin, M., Kleber, S., Dang, Y., Gülcüler Balta, G. S., Kalamakis, G., et al. (2019). Onset of differentiation is post-transcriptionally controlled in adult neural stem cells. Nature 566, 100-104. doi: 10.1038/s41586-019-0888-x

Bell, K. F. S., Al-Mubarak, B., Martel, M.-A., McKay, S., Wheelan, N., Hasel, P., et al. (2015). Neuronal development is promoted by weakened intrinsic antioxidant defences due to epigenetic repression of Nrf2. Nat. Commun. 6:7066. doi: 10. 1038/ncomms8066

Berg, D. A., Su, Y., Jimenez-Cyrus, D., Patel, A., Huang, N., Morizet, D., et al. (2019). A Common Embryonic Origin of Stem Cells Drives Developmental and Adult Neurogenesis. Cell 177, 654-668.e15. doi: 10.1016/j.cell.2019.02.010

Bhargava, V., Head, S. R., Ordoukhanian, P., Mercola, M., and Subramaniam, S. (2015). Technical Variations in Low-Input RNA-seq Methodologies. Sci. Rep. 4:3678. doi: $10.1038 /$ srep03678

Borrett, M. J., Innes, B. T., Jeong, D., Tahmasian, N., Storer, M. A., Bader, G. D., et al. (2020). Single-Cell Profiling Shows Murine Forebrain Neural Stem Cells Reacquire a Developmental State when Activated for Adult Neurogenesis. Cell Rep. 32:108022. doi: 10.1016/j.celrep.2020.108022

Butler, A., Hoffman, P., Smibert, P., Papalexi, E., and Satija, R. (2018). Integrating single-cell transcriptomic data across different conditions, technologies, and species. Nat. Biotechnol. 36, 411-420. doi: 10.1038/nbt.4096

DeLuca, D. S., Levin, J. Z., Sivachenko, A., Fennell, T., Nazaire, M.-D., Williams, C., et al. (2012). RNA-SeQC: RNA-seq metrics for quality control and process optimization. Bioinformatics 28, 1530-1532. doi: 10.1093/bioinformatics/ bts 196

Denninger, J. K., Chen, X., Turkoglu, A. M., Sarchet, P., Volk, A. R., Rieskamp, J. D., et al. (2020). Defining the adult hippocampal neural stem cell secretome: in vivo versus in vitro transcriptomic differences and their correlation to secreted protein levels. Brain Res. 1735:146717. doi: 10.1016/j.brainres.2020.146717

Denoth-Lippuner, A., and Jessberger, S. (2021). Formation and integration of new neurons in the adult hippocampus. Nat. Rev. Neurosci. 22, 223-236. doi: 10. 1038/s41583-021-00433-Z

Diril, M. K., Ratnacaram, C. K., Padmakumar, V. C., Du, T., Wasser, M., Coppola, V., et al. (2012). Cyclin-dependent kinase 1 (Cdk1) is essential for cell division and suppression of DNA re-replication but not for liver regeneration. Proc. Natl. Acad. Sci. U.S.A. 109, 3826-3831. doi: 10.1073/pnas.1115201109

Dulken, B. W., Buckley, M. T., Navarro Negredo, P., Saligrama, N., Cayrol, R., Leeman, D. S., et al. (2019). Single-cell analysis reveals T cell infiltration in old neurogenic niches. Nature 571, 205-210. doi: 10.1038/s41586-019-1362-5

Dulken, B. W., Leeman, D. S., Boutet, S. C., Hebestreit, K., and Brunet, A. (2017). Single-Cell Transcriptomic Analysis Defines Heterogeneity and Transcriptional Dynamics in the Adult Neural Stem Cell Lineage. Cell Rep. 18, 777-790. doi: 10.1016/j.celrep.2016.12.060
Finak, G., McDavid, A., Yajima, M., Deng, J., Gersuk, V., Shalek, A. K., et al. (2015). MAST: a flexible statistical framework for assessing transcriptional changes and characterizing heterogeneity in single-cell RNA sequencing data. Genome Biol. 16:278. doi: 10.1186/s13059-015-0844-5

Gupta, A. K., and Nadarajah, S. (2004). Handbook Of Beta Distribution And Its Applications, 1st Edn. Boca Raton: CRC Press.

Harris, L., Rigo, P., Stiehl, T., Gaber, Z. B., Austin, S. H. L., Masdeu, M., et al. (2021). Coordinated changes in cellular behavior ensure the lifelong maintenance of the hippocampal stem cell population. Cell Stem Cell 28, 863-876.e6. doi: 10.1016/ j.stem.2021.01.003

Harrow, J., Denoeud, F., Frankish, A., Reymond, A., Chen, C.-K., Chrast, J., et al. (2006). GENCODE: producing a reference annotation for ENCODE. Genome Biol. S4, 1-9.

Harrow, J., Frankish, A., Gonzalez, J. M., Tapanari, E., Diekhans, M., Kokocinski, F., et al. (2012). GENCODE: the reference human genome annotation for The ENCODE Project. Genome Res. 22, 1760-1774. doi: 10.1101/ gr.135350.111

Hochgerner, H., Zeisel, A., Lönnerberg, P., and Linnarsson, S. (2018). Conserved properties of dentate gyrus neurogenesis across postnatal development revealed by single-cell RNA sequencing. Nat. Neurosci. 21, 290-299. doi: 10.1038/ s41593-017-0056-2

Kawase, T., Ohki, R., Shibata, T., Tsutsumi, S., Kamimura, N., Inazawa, J., et al. (2009). PH Domain-Only Protein PHLDA3 Is a p53-Regulated Repressor of Akt. Cell 136, 535-550. doi: 10.1016/j.cell.2008.12.002

Kim, D., Langmead, B., and Salzberg, S. L. (2015). HISAT: a fast spliced aligner with low memory requirements. Nat. Methods 12, 357-360. doi: 10.1038/nmeth. 3317

Kjell, J., Fischer-Sternjak, J., Thompson, A. J., Friess, C., Sticco, M. J., Salinas, F., et al. (2020). Defining the Adult Neural Stem Cell Niche Proteome Identifies Key Regulators of Adult Neurogenesis. Cell Stem Cell 26, 277-293.e8. doi: 10.1016/j.stem.2020.01.002

Knight, V. B., and Serrano, E. E. (2018). Expression analysis of RNA sequencing data from human neural and glial cell lines depends on technical replication and normalization methods. BMC Bioinformatics 19:412. doi: 10.1186/s12859018-2382-0

Kroll, K. W., Mokaram, N. E., Pelletier, A. R., Frankhouser, D. E., Westphal, M. S., Stump, P. A., et al. (2014). Quality Control for RNA-Seq (QuaCRS): an Integrated Quality Control Pipeline. Cancer Inform. 13, 7-14. doi: 10.4137/CIN. S14022

Kuhn, R. M., Haussler, D., and Kent, W. J. (2013). The UCSC genome browser and associated tools. Brief. Bioinform. 14, 144-161. doi: 10.1093/bib/ bbs038

Kulkarni, A., Anderson, A. G., Merullo, D. P., and Konopka, G. (2019). Beyond bulk: a review of single cell transcriptomics methodologies and applications. Curr. Opin. Biotechnol. 58, 129-136. doi: 10.1016/j.copbio.2019.03.001

Law, C. W., Chen, Y., Shi, W., and Smyth, G. K. (2014). voom: precision weights unlock linear model analysis tools for RNA-seq read counts. Genome Biol. 15:R29. doi: 10.1186/gb-2014-15-2-r29

Liao, Y., Smyth, G. K., and Shi, W. (2013). The Subread aligner: fast, accurate and scalable read mapping by seed-and-vote. Nucleic. Acids. Res. 41, e108-e108. doi: 10.1093/nar/gkt214

Liao, Y., Smyth, G. K., and Shi, W. (2014). featureCounts: an efficient general purpose program for assigning sequence reads to genomic features. Bioinformatics 30, 923-930. doi: 10.1093/bioinformatics/btt656

Llorens-Bobadilla, E., Zhao, S., Baser, A., Saiz-Castro, G., Zwadlo, K., and Martin-Villalba, A. (2015). Single-Cell Transcriptomics Reveals a Population of Dormant Neural Stem Cells that Become Activated upon Brain Injury. Cell Stem Cell 17, 329-340. doi: 10.1016/j.stem.2015.07.002

Love, M. I., Huber, W., and Anders, S. (2014). Moderated estimation of fold change and dispersion for RNA-seq data with DESeq2. Genome Biol. 15:550. doi: 10.1186/s13059-014-0550-8

Marini, F., and Binder, H. (2019). pcaExplorer: an R/Bioconductor package for interacting with RNA-seq principal components. BMC Bioinformatics 20:331. doi: 10.1186/s12859-019-2879-1

McAvoy, K. M., and Sahay, A. (2017). Targeting Adult Neurogenesis to Optimize Hippocampal Circuits in Aging. Neurotherapeutics 14, 630-645. doi: 10.1007/ s13311-017-0539-6 
Mignone, J. L., Kukekov, V., Chiang, A.-S., Steindler, D., and Enikolopov, G. (2004). Neural stem and progenitor cells in nestin-GFP transgenic mice. J. Comp. Neurol. 469, 311-324. doi: 10.1002/cne.10964

Miller, S. M., and Sahay, A. (2019). Functions of adult-born neurons in hippocampal memory interference and indexing. Nat. Neurosci. 22, 1565-1575. doi: 10.1038/s41593-019-0484-2

Mizrak, D., Levitin, H. M., Delgado, A. C., Crotet, V., Yuan, J., Chaker, Z., et al. (2019). Single-Cell Analysis of Regional Differences in Adult V-SVZ Neural Stem Cell Lineages. Cell Rep. 26, 394-406.e5. doi: 10.1016/j. celrep.2018.12.044

Mou, T., Deng, W., Gu, F., Pawitan, Y., and Vu, T. N. (2020). Reproducibility of Methods to Detect Differentially Expressed Genes from SingleCell RNA Sequencing. Front. Genet. 10:1331. doi: 10.3389/fgene.2019. 01331

Nielsen, C. F., Zhang, T., Barisic, M., Kalitsis, P., and Hudson, D. F. (2020). Topoisomerase II $\alpha$ is essential for maintenance of mitotic chromosome structure. Proc. Natl. Acad. Sci. U.S. A. 117, 12131-12142. doi: 10.1073/pnas. 2001760117

O'Leary, N. A., Wright, M. W., Brister, J. R., Ciufo, S., Haddad, D., McVeigh, R., et al. (2016). Reference sequence (RefSeq) database at NCBI: current status, taxonomic expansion, and functional annotation. Nucleic. Acids Res. 44, D733D745. doi: 10.1093/nar/gkv1189

Qiu, X., Mao, Q., Tang, Y., Wang, L., Chawla, R., Pliner, H. A., et al. (2017). Reversed graph embedding resolves complex single-cell trajectories. Nat. Methods 14, 979-982. doi: 10.1038/nmeth.4402

Quinlan, A. R., and Hall, I. M. (2010). BEDTools: a flexible suite of utilities for comparing genomic features. Bioinformatics 26, 841-842. doi: 10.1093/ bioinformatics/btq033

Robinson, M. D., and Oshlack, A. (2010). A scaling normalization method for differential expression analysis of RNA-seq data. Genome Biol. 11:R25. doi: 10.1186/gb-2010-11-3-r25

Schubert, M., Lindgreen, S., and Orlando, L. (2016). AdapterRemoval v2: rapid adapter trimming, identification, and read merging. BMC Res. Notes 9:88. doi: 10.1186/s13104-016-1900-2

Shin, J., Berg, D. A., Zhu, Y., Shin, J. Y., Song, J., Bonaguidi, M. A., et al. (2015). Single-Cell RNA-Seq with Waterfall Reveals Molecular Cascades underlying Adult Neurogenesis. Cell Stem Cell 17, 360-372. doi: 10.1016/j.stem.2015.0 7.013

Soneson, C., and Robinson, M. D. (2018). Bias, robustness and scalability in singlecell differential expression analysis. Nat. Methods 15, 255-261. doi: 10.1038/ nmeth.4612

Squair, J. W., Gautier, M., Kathe, C., Anderson, M. A., James, N. D., Hutson, T. H., et al. (2021). Confronting false discoveries in single-cell differential expression. Nat. Commun. 12:5692. doi: 10.1038/s41467-021-25960-2

Stuart, T., Butler, A., Hoffman, P., Hafemeister, C., Papalexi, E., Mauck, W. M., et al. (2019). Comprehensive Integration of Single-Cell Data. Cell 177, 18881902.e21. doi: 10.1016/j.cell.2019.05.031

Sun, X., and Kaufman, P. D. (2018). Ki-67: more than a proliferation marker. Chromosoma 127, 175-186. doi: 10.1007/s00412-018-0659-8

Tapp, Z. M., Kumar, J. E., Witcher, K. G., Atluri, R. R., Velasquez, J. A., O’Neil, S. M., et al. (2020). Sleep Disruption Exacerbates and Prolongs the Inflammatory Response to Traumatic Brain Injury. J. Neurotrauma 37, 18291843. doi: $10.1089 /$ neu.2020.7010

Trapnell, C., Hendrickson, D. G., Sauvageau, M., Goff, L., Rinn, J. L., and Pachter, L. (2013). Differential analysis of gene regulation at transcript resolution with RNA-seq. Nat. Biotechnol. 31, 46-53. doi: 10.1038/nbt.2450
Urbán, N., Blomfield, I. M., and Guillemot, F. (2019). Quiescence of Adult Mammalian Neural Stem Cells: A Highly Regulated Rest. Neuron 104, 834-848. doi: 10.1016/j.neuron.2019.09.026

Vicidomini, C., Guo, N., and Sahay, A. (2020). Communication Cross Talk, and Signal Integration in the Adult Hippocampal Neurogenic Niche. Neuron 105, 220-235. doi: 10.1016/j.neuron.2019.11.029

Walker, L. A., Sovic, M. G., Chiang, C.-L., Hu, E., Denninger, J. K., Chen, X., et al. (2020). CLEAR: coverage-based limiting-cell experiment analysis for RNA-seq. J. Transl. Med. 18:63. doi: 10.1186/s12967-020-02247-6

Wang, L., Wang, S., and Li, W. (2012). RSeQC: quality control of RNA-seq experiments. Bioinformatics 28, 2184-2185. doi: 10.1093/bioinformatics/bts356

Wang, T., Li, B., Nelson, C. E., and Nabavi, S. (2019). Comparative analysis of differential gene expression analysis tools for single-cell RNA sequencing data. Bioinformatics 20:40. doi: 10.1186/s12859-019-2599-6

Yuzwa, S. A., Borrett, M. J., Innes, B. T., Voronova, A., Ketela, T., Kaplan, D. R., et al. (2017). Developmental Emergence of Adult Neural Stem Cells as Revealed by Single-Cell Transcriptional Profiling. Cell Rep. 21, 3970-3986. doi: 10.1016/ j.celrep.2017.12.017

Zeng, Z., Miao, N., and Sun, T. (2018). Revealing cellular and molecular complexity of the central nervous system using single cell sequencing. Stem Cell Res. Ther. 9:234. doi: 10.1186/s13287-018-0985-z

Zheng, G. X. Y., Terry, J. M., Belgrader, P., Ryvkin, P., Bent, Z. W., Wilson, R., et al. (2017). Massively parallel digital transcriptional profiling of single cells. Nat. Commun. 8:14049. doi: 10.1038/ncomms14049

Ziegenhain, C., Vieth, B., Parekh, S., Reinius, B., Guillaumet-Adkins, A., Smets, M., et al. (2017). Comparative Analysis of Single-Cell RNA Sequencing Methods. Mol. Cell 65, 631-643.e4. doi: 10.1016/j.molcel.2017.01.023

Zywitza, V., Misios, A., Bunatyan, L., Willnow, T. E., and Rajewsky, N. (2018). Single-Cell Transcriptomics Characterizes Cell Types in the Subventricular Zone and Uncovers Molecular Defects Impairing Adult Neurogenesis. Cell Rep. 25, 2457-2469.e8. doi: 10.1016/j.celrep.2018.11.003

Conflict of Interest: The authors declare that the research was conducted in the absence of any commercial or financial relationships that could be construed as a potential conflict of interest.

Publisher's Note: All claims expressed in this article are solely those of the authors and do not necessarily represent those of their affiliated organizations, or those of the publisher, the editors and the reviewers. Any product that may be evaluated in this article, or claim that may be made by its manufacturer, is not guaranteed or endorsed by the publisher.

Citation: Denninger JK, Walker LA, Chen X, Turkoglu A, Pan A, Tapp Z, Senthilvelan S, Rindani R, Kokiko-Cochran ON, Bundschuh $R$, Yan $P$ and Kirby ED (2022) Robust Transcriptional Profiling and Identification of Differentially Expressed Genes With Low Input RNA Sequencing of Adult Hippocampal Neural Stem and Progenitor Populations. Front. Mol. Neurosci. 15:810722. doi: $10.3389 /$ fnmol.2022.810722

Copyright (c) 2022 Denninger, Walker, Chen, Turkoglu, Pan, Tapp, Senthilvelan, Rindani, Kokiko-Cochran, Bundschuh, Yan and Kirby. This is an open-access article distributed under the terms of the Creative Commons Attribution License (CC BY). The use, distribution or reproduction in other forums is permitted, provided the original author(s) and the copyright owner(s) are credited and that the original publication in this journal is cited, in accordance with accepted academic practice. No use, distribution or reproduction is permitted which does not comply with these terms. 